\title{
Synthetic tracheal grafts seeded with bone marrow cells fail to generate functional tracheae: First long-term follow-up study
}

\author{
Thomas Fux, MD, PhD, ${ }^{\mathrm{a}, \mathrm{b}}$ Cecilia Österholm, PhD, ${ }^{\mathrm{a}}$ Raquel Themudo, MD, PhD, ${ }^{\mathrm{a}, \mathrm{c}}$ \\ Oscar Simonson, MD, PhD, ${ }^{\mathrm{d}, \mathrm{e}}$ Karl-Henrik Grinnemo, MD, PhD, ${ }^{\mathrm{a}, \mathrm{d}, \mathrm{e}}$ and Matthias Corbascio, MD, $\mathrm{PhD}^{\mathrm{a}, \mathrm{f}}$
}

\section{ABSTRACT}

Objective: Synthetic tracheal grafts seeded with autologous bone marrowmononuclear cells (BM-MNCs) have been described as becoming living and functional grafts representing a promising option for tracheal replacement for pathologies unamenable by segmental resection or autologous repair. This study aimed to present the first long-term follow-up of these procedures in humans.

Methods: We retrospectively analyzed 3 patients who received synthetic tracheal grafts seeded with BM-MNCs implanted.

Results: Patient 1 was a 37-year-old man with mucoepidermoid carcinoma, the first-ever human to receive a synthetic tracheal graft seeded with BM-MNCs. Patient 2 was a 30-year-old man with adenoid cystic carcinoma, and patient 3 was a 22-year-old woman with an iatrogenic tracheal injury. All patients developed graft-related complications necessitating multiple surgical reinterventions. Patient 1 was hospitalized for 8 months before dying from respiratory failure secondary to graft dehiscence 32 months after implantation. Patient 2 died 3.5 months after implantation from undisclosed causes. Patient 3 received a second synthetic tracheal graft after 11 months and an allogeneic trachea and lung transplantation 45 months after the primary implantation. Patient 3 underwent 191 surgical interventions after the primary implantation and spent 55 months in the intensive care unit before dying from airway bleeding. All patients' bronchoscopic, histologic, and radiologic investigations demonstrated graft-associated complications, including anastomotic fistulae and obstructive granulation tissue, without graft vascularization, mucosal lining, or integration into adjacent tissues.

Conclusions: Synthetic tracheal grafts seeded with BM-MNCs do not become living functional tracheal grafts and lead to debilitating complications and death. ( $\mathrm{J}$ Thorac Cardiovasc Surg 2020;159:2525-37)

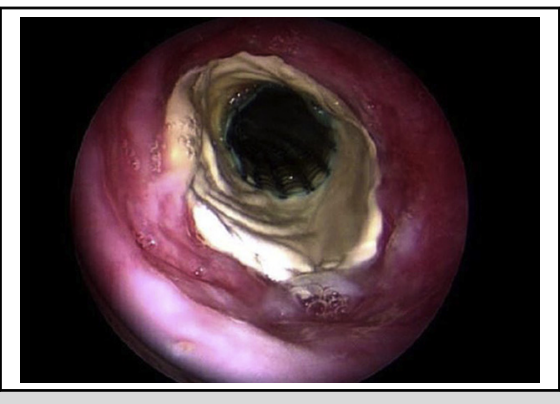

Patient 3: Bronchoscopy 6.5 months after implantation: graft without mucosal lining.

\section{CENTRAL MESSAGE}

Synthetic tracheal grafts seeded with BM-MNCs fail to become living, functional tracheal grafts and lead to severe complications and death.

\section{PERSPECTIVE}

Synthetic tracheal grafts seeded with BM-MNCs have been described to generate living and functional grafts that integrate into adjacent tissues. This first long-term follow-up study of implantations in humans presents contrary data. Further preclinical studies are needed to address basic biological concepts before further implantations are performed.

See Commentaries on pages 2537,2539 , and 2541.
The trachea has a complex anatomy and physiology serving as a mechanically stable, open, and airtight conduit for ventilation between the larynx and the main bronchi. It

\footnotetext{
From the ${ }^{\text {a }}$ Department of Molecular Medicine and Surgery, Karolinska Institutet, Stockholm; 'bection Cardiothoracic Surgery and Anesthesiology, Division of Perioperative Medicine and Intensive Care, ${ }^{\mathrm{c}}$ Division of Radiology, and ${ }^{\mathrm{f}} \mathrm{Heart}$ and Vascular Division, Karolinska University Hospital, Stockholm; ${ }^{\mathrm{d}}$ Department of Surgical Sciences, Uppsala University, Uppsala; and ${ }^{\mathrm{e}}$ Department of Cardiothoracic Surgery and Anesthesiology, Uppsala University Hospital, Uppsala, Sweden.

Received for publication July 8, 2019; revisions received Sept 19, 2019; accepted for publication Sept 26, 2019; available ahead of print Dec 16, 2019.
}

humidifies the air and facilitates clearance of microbeand toxin-contaminated tracheobronchial secretions. ${ }^{1,2}$ The trachea depends on segmental arteries for its blood

\footnotetext{
Address for reprints: Thomas Fux, MD, PhD, Section Cardiothoracic Surgery and Anesthesiology, Division of Perioperative Medicine and Intensive Care, Karolinska University Hospital, SE-171 76 Stockholm, Sweden (E-mail: thomas. fux@sll.se).

0022-5223

Copyright (C) 2019 by The American Association for Thoracic Surgery. Published by Elsevier Inc. This is an open access article under the CC BY-NC-ND license (http:// creativecommons.org/licenses/by-nc-nd/4.0/).

https://doi.org/10.1016/j.jtcvs.2019.09.185
} 


\section{Abbreviations and Acronyms}

BM-MNCs $=$ bone marrow-mononuclear cells

$\mathrm{CT}=$ computed tomography

$\mathrm{KUH}=$ Karolinska University Hospital

PET = polyethylene terephthalate

$\mathrm{PET} / \mathrm{PU}=$ polyethylene terephthalate and polyurethane

TGF- $\beta 3=$ transforming growth factor $-\beta 3$

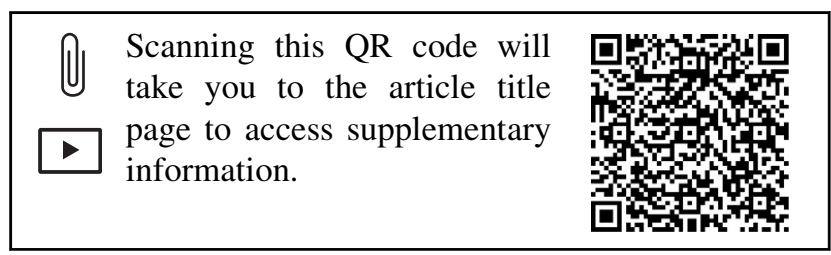

supply, branching into an intricate network of small vessels that reach into the endotracheal mucosal lining to support specialized epithelium. ${ }^{2-5}$ Different strategies have been developed to treat segmental tracheal pathologies. ${ }^{1,4,6-10}$ In adults, tracheal resection with end-to-end anastomosis may be considered for tracheal pathologies extending for up to half of the tracheal length. For more extensive pathologies, tracheal reconstruction or replacement is required. ${ }^{6,9}$ The major challenges in tracheal replacement are vascularization and the permanent interface between the graft and the microbe-contaminated lumen of the respiratory tract. ${ }^{1,4,10-13}$ Without revascularization, the graft with its specialized epithelium cannot survive or integrate into adjacent tissues and the barrier function to the outside world will be lost, resulting in chronic infection. Shortage of donor organs and the need for immunosuppression are further concerns when considering allogeneic tracheal transplantation.,

Synthetic tracheal grafts seeded with autologous bone marrow-mononuclear cells (BM-MNCs) in combination with locally and systemically applied growth factors have been described to generate living, functional grafts with sitespecific epithelialization that integrate into adjacent tissues. ${ }^{14-17}$ This would represent a paradigm shift not just within tracheal surgery and organ transplantation but also within the field of regenerative medicine by circumventing the need for immunosuppression and allow for rapid manufacturing of tailor-made organs. However, the validity of the claimed success of these procedures has been challenged and subsequently found to be tainted with scientific misconduct and lacking both ethical and regulatory approval, ie, the Regional Ethical Review Board in Stockholm did not approve an application for these implantations. ${ }^{7,18-28}$ Despite that more than 10 implantations in humans have been performed in Sweden, Russia, the United States, and the
United Kingdom, a paucity of follow-up data remains. The aim of this study was to present the first long-term follow-up of 3 patients who in total received 4 synthetic tracheal grafts seeded with BM-MNCs.

\section{MATERIALS AND METHODS}

We retrospectively reviewed the medical records, including all recorded bronchoscopies and histologic and radiologic investigations of 3 patients who received 4 synthetic tracheal grafts seeded with BM-MNCs implanted between June 2011 and July 2013 at the Karolinska University Hospital (KUH), Stockholm, Sweden. The Regional Ethical Review Board in Stockholm approved this study (IRB approval 2014/1532-31/1).

\section{RESULTS \\ Patient 1}

The first patient to ever receive a BM-MNC-seeded synthetic tracheal graft was a 37-year-old man with low-grade mucoepidermoid carcinoma of the trachea who was treated with debulking surgery and radiation in 2009. ${ }^{14,29}$ A recurrence of the primary malignancy was suspected in early 2011, and the patient was referred to KUH for clinical evaluation and synthetic trachea implantation. On admission in May 2011, the patient was asymptomatic. Preoperative computed tomography (CT) scan revealed a distal tracheal obstruction of $25 \%$ with a tumor mass measuring 9-mm craniocaudal $\times 7$-mm intraluminal $\times 10$-mm cross-sectional diameter (Figure 1, A).

The decision was made to replace the lower trachea and proximal parts of the main bronchi with a tracheobronchial graft made of the nanocomposite polymer POSS-PCU (polyhedral oligomeric silsesquioxane covalently bonded to poly [carbonate-urea] urethane; University College of London, London, United Kingdom) (Table 1). A bone marrow aspiration was performed to retrieve autologous BM-MNCs that were seeded onto the synthetic graft in a rotating bioreactor (Harvard Apparatus Regenerative Technology, Holliston, Mass) for 36 hours before implantation in June 2011. 14,15,17 Immediately before implantation, a second bone marrow harvest was performed and the graft was reseeded with BM-MNCs and conditioned with recombinant human transforming growth factor- $\beta 3$ (TGF- $\beta 3$; R\&D Systems, Minneapolis, Minn; $10 \mu \mathrm{g} / \mathrm{cm}^{2}$ ), granulocyte colonystimulating factor filgrastim (Neupogen, Amgen Europe BV, Breda, The Netherlands; $10 \mu \mathrm{g} / \mathrm{kg}$ ), and epoetin- $\beta$ (synthetic erythropoietin; Roche, Grenzach-Wyhlen, Germany; 40,000 UI) (Table 1). ${ }^{14}$

In addition, off-label supratherapeutic doses of granulocyte colony-stimulating factor filgrastim $(10 \mu \mathrm{g} / \mathrm{kg}$ every other day for 10 days) and epoetin- $\alpha(40,000$ IU every other day for 12 days) were postoperatively administered to the patient as a "regenerative boosting therapy" to enhance a generative process of the nanocomposite to become a "living and functional graft" (Table 1). ${ }^{14}$ However, the 7 control biopsies retrieved from the graft immediately before 

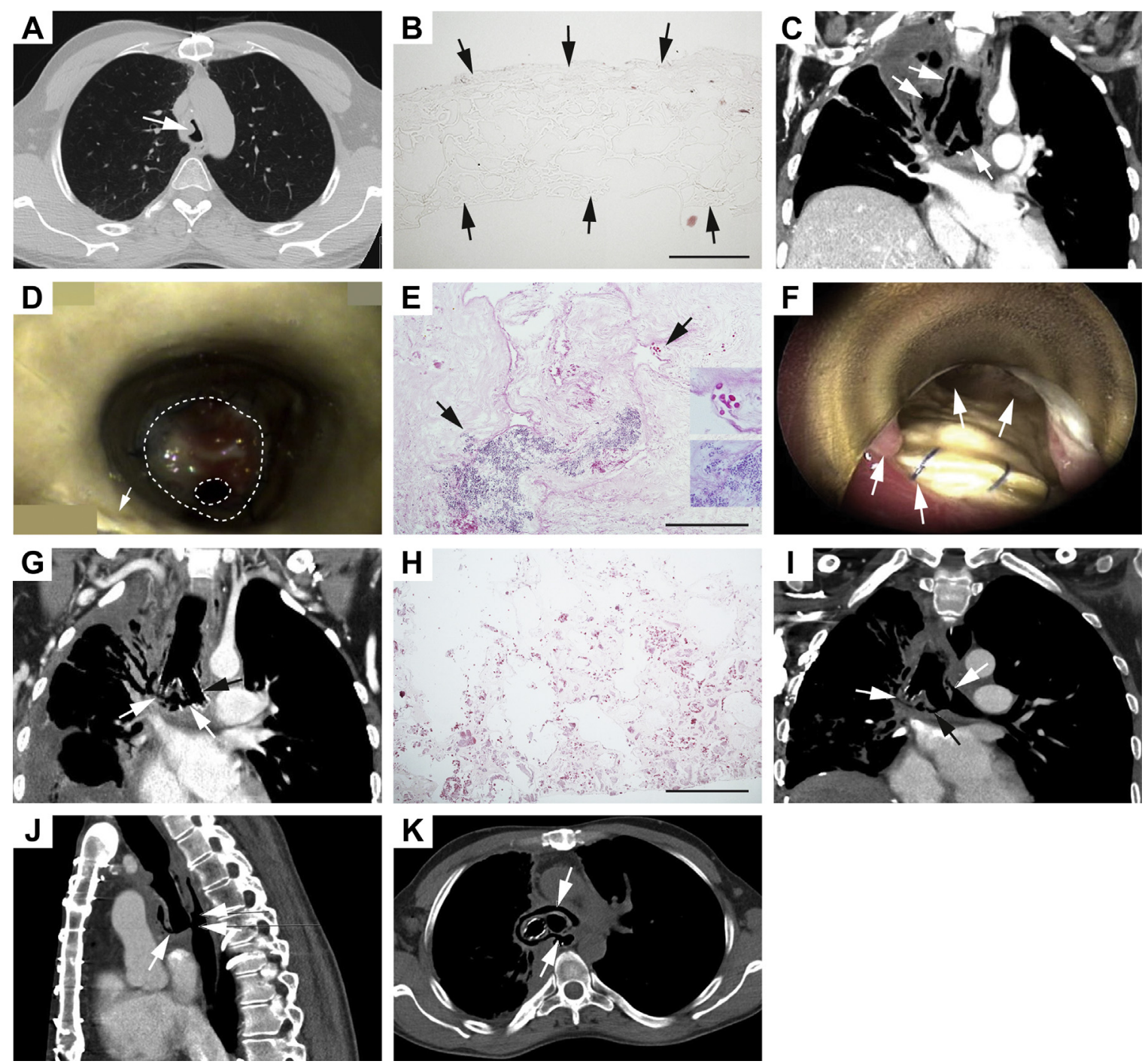

FIGURE 1. Bronchoscopic, radiologic, and histologic findings in patient 1. A, Preoperative computed tomography (CT) scan of the chest 17 days before graft implantation. Arrow: tracheal tumor measuring 7-mm intraluminal $\times 10-\mathrm{mm}$ crossectional diameter (9-mm craniocaudal diameter not shown in this image), obstructing $25 \%$ of the distal tracheal lumen. B, Biopsy from the synthetic graft at the time of graft implantation. Porous synthetic material (the borders of the synthetic graft are outlined by black arrows) without detectable cellular components, layer or matrix structures. Hematoxylin and eosin (H\&E) staining $\times 10$ (scale bar $=100 \mu \mathrm{m})$. C, CT scan of the chest 4 weeks after graft implantation. Left two upper arrows: air outside the graft. Right arrow: fistula at the left distal graft anastomosis. Anastomotic fistula and graft in contact with ambient air indicate graft infection and inability of the synthetic graft to integrate into adjacent tissues. D, Bronchoscopy 2.5 months after graft implantation. Inner graft wall without vascularization and mucosal lining. Arrow: graft carina. Large dashed circle: right distal graft ending. Small dashed circle: native right main bronchus opening obstructed with granulation tissue (opening expected to have the same area as the distal graft ending). E, Biopsy from the distal graft anastomosis 2.5 months after graft implantation. Granulation tissue with necrosis and infection. Left arrow: bacterial colonies. Right arrow: fungal hyphae. Gram and periodic acid-Schiff staining $\times 20$ (scale bar $=100 \mu \mathrm{m}$ ). F, Bronchoscopy 5.5 months after graft implantation. Proximal graft anastomosis and the synthetic graft. Inner wall of the bronchoscope in the upper part of the image. Absence of mucosal lining on the inner wall of the yellow-white-colored graft. Arrows from left to right: anastomotic granulation tissue, exposed suture at the proximal graft anastomosis, and the left and right distal graft departures. G, CT scan of the chest 5.5 months after graft implantation. Left white arrow: fistula at the right distal graft anastomosis. Right white arrow: fistula at left distal graft anastomosis. Black arrow: airway stent in the left distal graft departure and the left main bronchus. Airway stent visible in the right graft departure. H, Biopsy from the inner graft wall above the carina 11.5 months after graft implantation. Acellular structures and graft remnants. Mucosal lining could not be identified. H\&E staining (scale bar $=100 \mu \mathrm{m}$ ). I, CT of the chest 28 months after graft implantation. Graft detached and dislocated from adjacent tissues. Left white arrow and black arrow: right and left distal anastomotic dehiscence. Right white arrow: dislocated graft with air outside the graft indicating remaining absence of integration into adjacent tissues 28 months after implantation. J, CT scan of the chest 28 months after graft implantation. Synthetic graft detached and dislocated from adjacent tissues. Left arrow: distal graft detachment with tracheomediastinal fistula. Right upper and lower arrows: boundaries of the ventral esophageal wall defect (tracheoesophageal fistula). K, CT scan of the chest 28 months after graft implantation. Upper arrow: graft not being attached to adjacent tissues and surrounded by air. Lower arrow: esophagomediastinal fistula. Air surrounding the synthetic graft and tracheoesophageal fistulae indicate graft contamination by air and secretions from the airways and the esophagus. The right distal anastomotic-mediastinoesophageal fistula can be anticipated in the right distal graft departure identified by the visible (white-colored) airway stent. 
TABLE 1. Patient and synthetic tracheal graft characteristics

\begin{tabular}{|c|c|c|c|}
\hline Characteristics & Patient 1 & Patient 2 & Patient 3 \\
\hline Sex & Male & Male & Female \\
\hline Age, y & 37 & 30 & 22 \\
\hline $\begin{array}{l}\text { Etiology of tracheal } \\
\text { disorder }\end{array}$ & $\begin{array}{l}\text { Relapse of low-grade* mucoepidermoid } \\
\text { carcinoma } \\
\text { No metastases }\end{array}$ & $\begin{array}{l}\text { Adenoid cystic carcinoma } \\
\text { No metastases }\end{array}$ & $\begin{array}{l}\text { First implantation: iatrogenic tracheal } \\
\text { injury } \\
\text { Second implantation: dysfunctional first } \\
\text { graft }\end{array}$ \\
\hline Status at admission & Asymptomatic, living at home & Asymptomatic, living at home & $\begin{array}{l}\text { Intermittent coughing, pleural drainage, } \\
\text { living at home }\end{array}$ \\
\hline $\begin{array}{l}\text { Preoperative tracheal } \\
\text { obstruction }\end{array}$ & $\begin{array}{l}25 \% \text { of the distal part of the } \\
\text { supracarinal trachea } \dagger\end{array}$ & None & None \\
\hline Urgency of surgery & Elective & Elective & Elective \\
\hline Graft material & $\begin{array}{l}\text { Nanocomposite polymer } \\
\text { of POSS-PCU }\end{array}$ & $\begin{array}{l}\text { Electrospun polyblend of PET/PU } \\
\quad 70 \% / 30 \%\end{array}$ & $\begin{array}{l}\text { First graft: electrospun polyblend of } \\
\text { PET/PU } 70 \% / 30 \% \\
\text { Second graft: electrospun PET } 100 \%\end{array}$ \\
\hline Manufacturer & $\begin{array}{l}\text { Seifalian, Macchiarini, University } \\
\text { College of London, } \\
\text { London, United Kingdom }\end{array}$ & Nanofiber Solutions, Hilliard, Ohio & $\begin{array}{l}\text { First graft: Nanofiber Solutions } \\
\text { Second graft: Harvard Apparatus } \\
\text { Regenerative Technology, HART, } \\
\text { Holliston, Mass }\end{array}$ \\
\hline $\begin{array}{l}\text { Substances associated } \\
\text { with the synthetic graft } \ddagger\end{array}$ & $\begin{array}{l}\text { TGF- } \beta 3 \\
\text { G-CSF (filgrastim) } \\
\text { Epoetin }\end{array}$ & $\begin{array}{l}\text { TGF- } \beta 3 \\
\text { G-CSF (filgrastim) } \\
\text { Epoetin }\end{array}$ & $\begin{array}{l}\text { TGF- } \beta 3 \\
\text { G-CSF (filgrastim) } \\
\text { Epoetin }\end{array}$ \\
\hline
\end{tabular}

POSS-PCU, Polyhedral oligomeric silsesquioxane-poly-[carbonate-urea] urethane; $P E T$, polyethylene terephthalate; $P U$, polyurethane; $T G F-\beta 3$, transforming growth factor $\beta-3$; $G$ - CSF, granulocyte-colony stimulating factor. *Brandwein classification. $\dagger$ The tumor mass measuring 9-mm craniocaudal $\times 7$-mm intraluminal $\times 10$-mm cross-sectional diameters. ‡Off-label and high-dose administration of specific substances immediately before and after implantation of the synthetic tracheal graft: conditioning of the graft in the bioreactor immediately before implantation with recombinant human TGF- $\beta 3$ (R\&D Systems, Minneapolis, Minn; $10 \mu \mathrm{g} / \mathrm{kg} \mathrm{cm}{ }^{2}$ ), G-CSF (filgastrim; Neupogen, Amgen Europe BV, Breda, Netherlands; $10 \mu \mathrm{g} / \mathrm{kg}$ ), and epoetin- $\beta$ (synthetic analogue of erythropoietin; Roche, Grenzach-Wyhlen, Germany; 40.000 IU). Postoperative administration as "regenerative boosting therapy": G-CSF $10 \mu \mathrm{g} / \mathrm{kg}$ every other day; in patient 1 for 10 days, in patient 2 for 12 days, and in patient 3 for 16 days after the first graft implantation and for 14 days after the second graft implantation, epoetin- $\alpha 40.000$ IU every other day; in patient 1 for 12 days, in patient 2 for 12 days, and in patient 3 for 16 days after the first graft implantation and for 14 days after the second graft implantation.

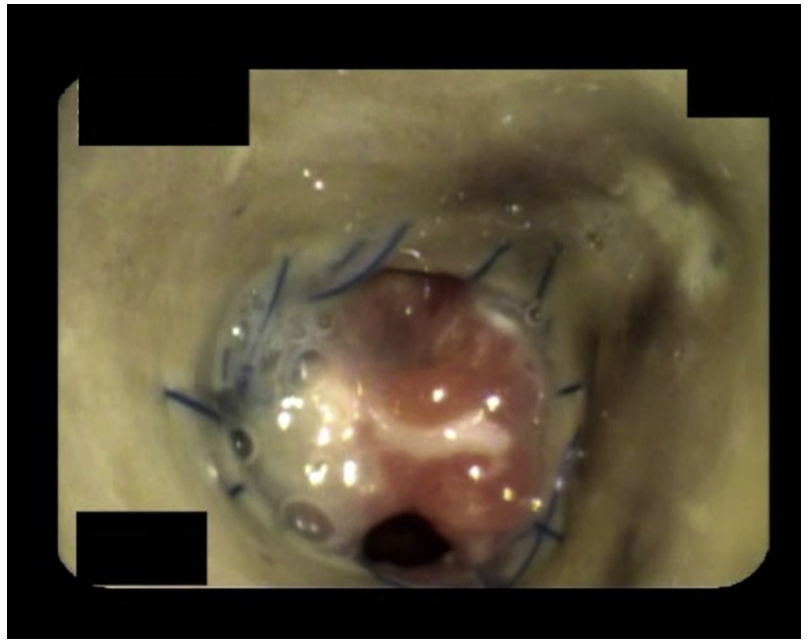

VIDEO 1. Patient 1. Bronchoscopy 2.5 months after implantation. Video available at: https://www.jtcvs.org/article/S0022-5223(19)32371-2/ fulltext.

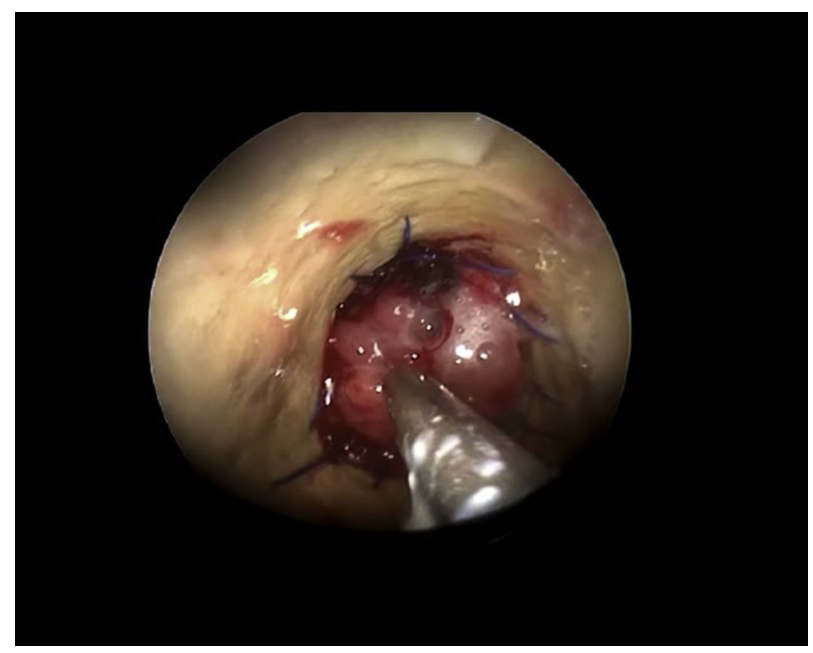

VIDEO 2. Patient 1. Bronchoscopy 5.5 months after implantation. The normal native trachea and the yellow-white-colored synthetic graft with obstructing granulation tissue, exposed sutures and fistulae at the anastomotic sites, and absence of mucosal lining on the inner graft wall. Video available at: https://www.jtcvs.org/article/S0022-5223(19)32371-2/ fulltext. 
TABLE 2. Adverse events, interventions, and outcomes of special interest

\begin{tabular}{|c|c|c|c|}
\hline Adverse events, interventions, and outcomes & Patient 1 & Patient 2 & Patient 3 \\
\hline \multicolumn{4}{|l|}{ Adverse events } \\
\hline \multicolumn{4}{|l|}{ Synthetic tracheal graft } \\
\hline Graft contamination by ambient air and oropharyngeal secretions & + & + & + \\
\hline Bacterial and fungal colonization (chronic graft infection) $\ddagger$ & + & + & + \\
\hline Graft obstruction (stagnation of mucus, blood, pus) & + & " & + \\
\hline Near-fatal suffocation events, $\mathrm{n}$ & + & ". & 40 \\
\hline Graft collapse (synthetic material fatigue) & - & ." & + \\
\hline \multicolumn{4}{|l|}{ Graft anastomoses } \\
\hline Graft-mediastinal and/or pleural fistula & + & + & + \\
\hline Graft-esophageal fistula & + & ". & + \\
\hline Massive air leak $\S$ & " & ." & + \\
\hline Excessive formation of obstructive granulation tissue & + & ". & + \\
\hline Granulation bleeding (multiple life-threatening hemoptysis) & - & ". & + \\
\hline Anastomotic wound breakdown (graft dehiscence) & + & ". & + \\
\hline Dislocation of graft and graft-bronchial airway stent, numerous & + & ". & + \\
\hline Mediastinitis & + & " & + \\
\hline Thromboembolism while on "regenerative boosting therapy" & + & + & + \\
\hline \multicolumn{4}{|l|}{ Interventions secondary to graft dysfunction } \\
\hline Resection of obstructive anastomotic granulation tissue (numerous) & + & .. & + \\
\hline Rigid bronchoscopy (airway stent evaluation and/or intervention), $\mathrm{n}$ 『 & 13 & ". & 56 \\
\hline Chronic tracheostomy (for airway clearance) & - & ". & + \\
\hline Daily scheduled and emergency flexible bronchoscopy, $\mathrm{n} \#$ & - & - & 7602 \\
\hline Open standing thoracotomy, chronic chest drainage & - & - & + \\
\hline Implantation of second synthetic tracheal graft & - & - & 1 \\
\hline Thoracic surgical intervention (sternotomy) & - & - & 3 \\
\hline Right-sided thoracoplasty (resection of ribs no. 2-8) & - & - & 1 \\
\hline Esophageal stenting, transection, esophagectomy, reconstruction ${ }^{* *}$ & + & - & + \\
\hline Ventilator support, $d$ & ". & ". & 305 \\
\hline Extracorporeal membrane oxygenation, days, $\mathrm{n} \dagger \dagger$ & - & - & 72 \\
\hline Hemodialysis, wk & .. & - & 7 \\
\hline
\end{tabular}

\section{Outcome}

Synthetic tracheal graft

Generation into a living and functional graft

Vascularization, mucosal lining, site-specific epithelialization

Integration into adjacent tissues

Allogeneic tracheal and single lung transplantation

Surgical reinterventions

Hospitalization, months $\ddagger \ddagger$

Survival after implantation, mo

Cause of death

Inability to ventilate (dysfunctional and dehiscent graft)

Undisclosed cause

Airway bleeding

$\begin{array}{llc}\text { No } & \text { No } & \text { No } \\ \text { No } & \text { No } & \text { No } \\ \text { No } & \text { No } & \text { No } \\ \text { No } & \text { No } & \text { Yes } \\ 16 & 6 & 191 \\ 12 & 2 & 56 \\ 32 & 3.5 & 55 \\ & & \\ + & . \cdot & . \\ . \cdot & + & . \\ . \cdot & \cdot \cdot & +\end{array}$

*See Table E1 for the full listing of adverse events and interventions of special interest. Number of adverse events, interventions and outcomes for all 3 patients are restricted to the time of hospitalization at Karolinska University Hospital and the information provided in the medical records at Karolinska University Hospital, as complete data have not been accessible from the healthcare providers abroad. The plus sign $(+)$ denotes presence of the variable but not the number. The minus sign $(-)$ denotes absence of the variable. Two mid-dots (") denote no available or not applicable data. †Data presented for patient 3 includes data after the first and second graft implantations. $\ddagger$ Multiresistant strepthomonas multi. (Stenothropomonas malthiphilia) in patient 1 and multiresistant Pseudomonas aeruginosa in patient 3. §Massive air leak trough anastomotic fistulae and out into room air by the open thoracotomy (open standing due to chronic thoracotomy cavity infection and pus accumulation). "Thromboembolism while on the "regenerative boosting therapy." Patient 1: pulmonary artery thrombus, patient 2: pulmonary artery, jugular, subclavian, and axillary vein thrombi and patient 3 after the second graft implantation: femoral artery and extracorporeal membrane oxygenation pump thrombosis. \Manufacturing of tailor-made airway stents (Leufen Medical, Berlin, Germany) for patient 3. \#Flexible bronchoscopies scheduled every fourth hour (6-15 per day) due to the 2 dysfunctional grafts in patient 3 . From implantation of the first graft until the patient was transferred to the thoracic transplant center after 3 years and 1 month. **Esophageal transection and subcutaneous colon interponate (patient 1). Esophagectomy and (failed) esophageal reconstruction by concomitant gastric pull up at the allogeneic tracheal and single lung transplantation (patient 3). $\dagger \dagger$ Insecure airway, massive anastomotic air leak, obstructing

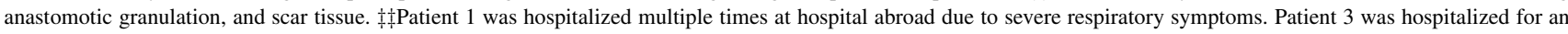
additional 1 year and 7 months after transfer to a thoracic transplant center. 


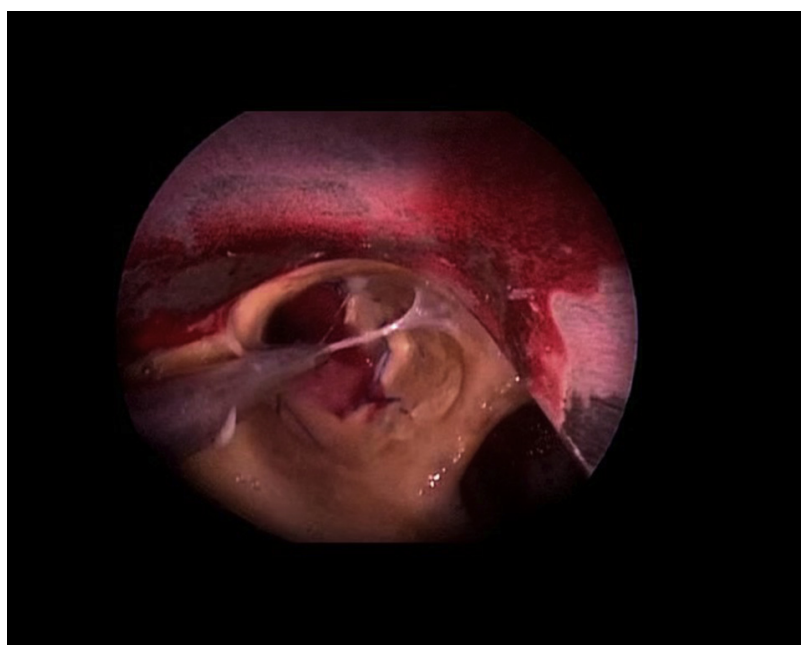

VIDEO 3. Patient 1. Bronchoscopy 11.5 months after implantation. Video available at: https://www.jtcvs.org/article/S0022-5223(19)32371-2/fulltext.

implantation could not provide any evidence of a cellular layer covering the graft (Figure 1, B).

CT scan at 4 weeks after implantation showed a fistula at the left bronchial anastomosis (Figure 1,C). Bronchoscopy performed after 2.5 months demonstrated a graft without mucosal lining, obstruction of the right distal anastomosis, and a left distal anastomotic fistula (Figure 1, D, Video 1, and Figures E1 and E2, $A$ and $B$ ). Biopsies from this time point could not demonstrate any mucosal lining on the graft but only bacterial and fungal colonization and granulation tissue and necrosis at the anastomotic sites (Figure 1,E).

Five and a half months after implantation, the patient had developed respiratory symptoms and was referred back to KUH for evaluation. At readmission, the patient had dyspnea, large amounts of sputum, absent right-sided respiratory sounds, and an involuntary loss of body weight of 11

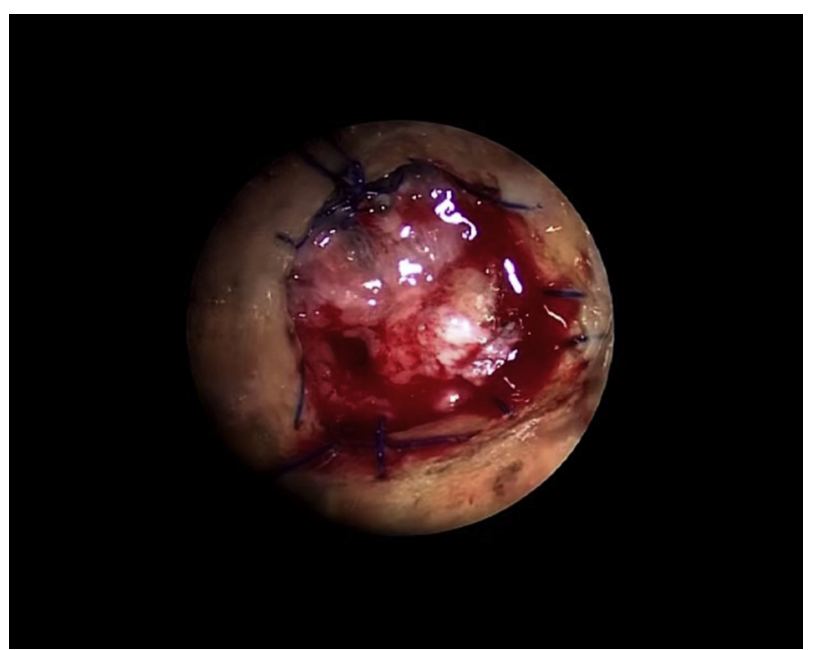

VIDEO 4. Patient 1. Bronchoscopy 17 months after implantation. Video available at: https://www.jtcvs.org/article/S0022-5223(19)32371-2/fulltext.

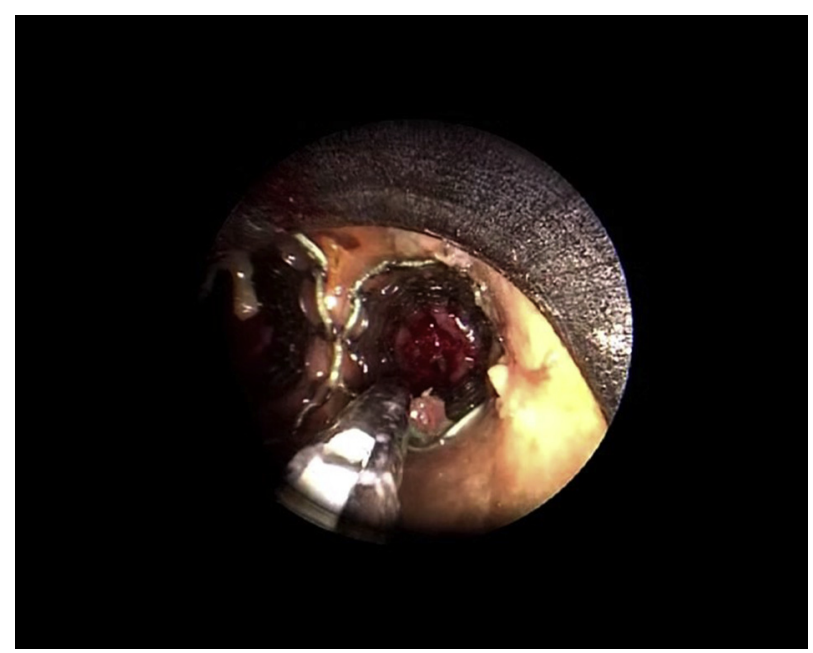

VIDEO 5. Patient 1. Bronchoscopy 22 months after implantation. Video available at: https://www.jtcvs.org/article/S0022-5223(19)32371-2/fulltext.

$\mathrm{kg}(81$ to $70 \mathrm{~kg})$. Bronchoscopy and CT scan revealed graftrelated complications with absent mucosal lining, total obstruction of the right bronchus anastomosis, and bilateral distal anastomotic fistulae with the graft being detached from the right bronchus when manipulated with the bronchoscope (Figure 1, $F$ and $G$, Video 2, and Figures E3, $A$, to E5, C). Repetitive resections of obstructive anastomotic granuloma and numerous stent interventions were initiated to seal the anastomotic fistulae and prevent graft detachment (Table 2). Bronchoscopy at 8 and 11.5 months and graft biopsy at 11.5 months could not identify any mucosal lining, only acellular structures and graft remnants (Figure 1, $H$, Video 3, and Figures E6, $A$, to E7, C).

The stent interventions brought only partial and temporary effect. The patient continued to deteriorate, suffering from aggravating dyspnea, multiple episodes

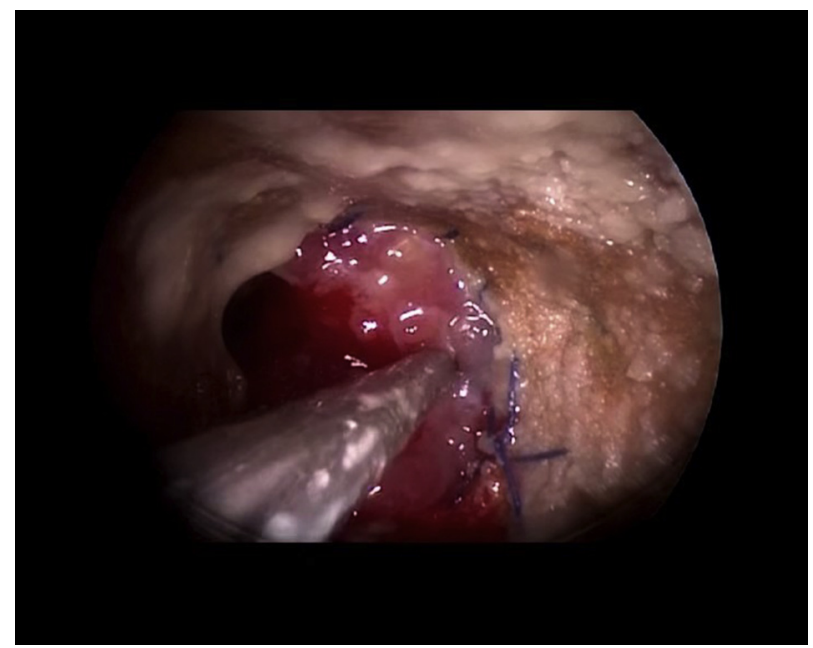

VIDEO 6. Patient 1. Bronchoscopy 27 months after implantation. Video available at: https://www.jtcvs.org/article/S0022-5223(19)32371-2/fulltext. 


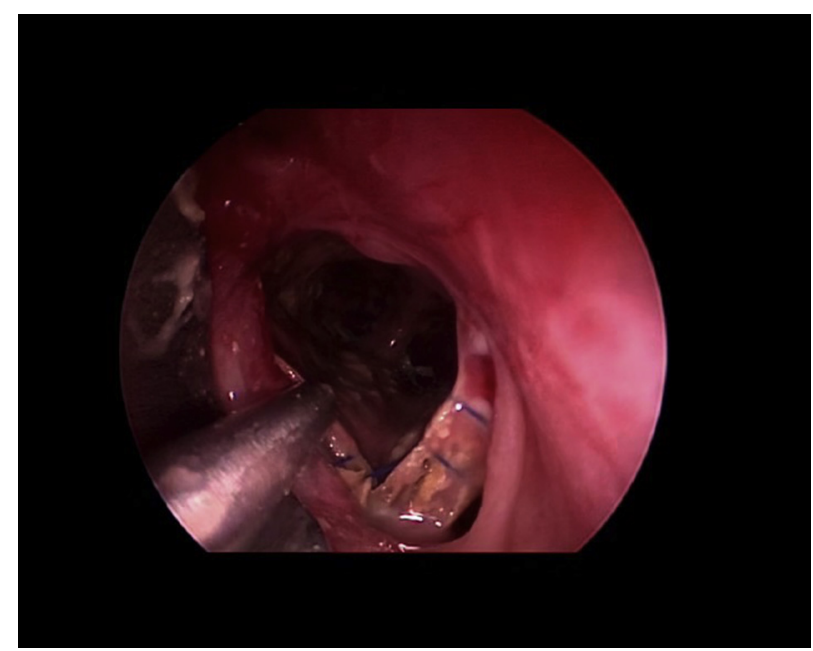

VIDEO 7. Patient 1. Bronchoscopy 29 months after implantation. Video available at: https://www.jtcvs.org/article/S0022-5223(19)32371-2/fulltext.

with copious hemoptysis, recurrent right-sided pneumonia, and a lung abscess. Investigations revealed a nonfunctioning right lung. A large tracheomediastinal-esophageal fistula was subsequently diagnosed requiring esophageal stenting followed by transection, as the planned esophagectomy would have dislodged the dehiscent graft (Figure $1, I$ to $K$ ). The patient was hospitalized the last 8 months of his life and suffocated due to graft detachment in January 2014, 32 months after implantation (Table 2). The autopsy revealed a completely detached synthetic graft without vascularization or mucosal lining surrounded by mediastinal abscess formation, chronic pericarditis, and right-sided pulmonary fibrosis (Table E1, which contains the full list of adverse events and interventions).

All bronchoscopies between 2.5 and 32 months after implantation $(2.5,5.5,6.5,8,11.5,17,22,27,29$, and

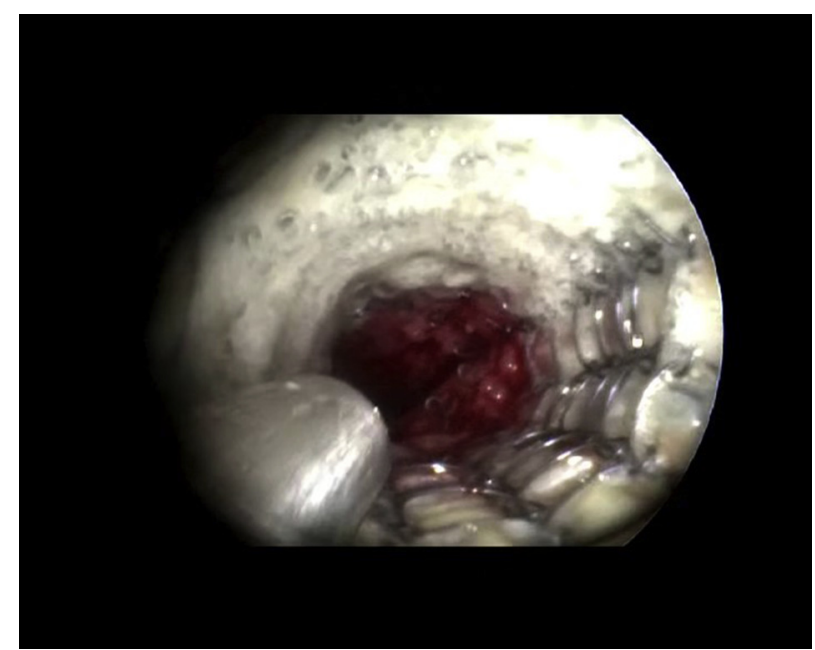

VIDEO 8. Patient 1. Bronchoscopy 32 months after implantation. Video available at: https://www.jtcvs.org/article/S0022-5223(19)32371-2/fulltext.
32 months) demonstrated graft-associated complications including anastomotic fistulae, obstructing granulation tissue, and absent mucosal lining, as exemplified by Videos 1 to 8, and Figures E1 to E11. All biopsies from the graft and the anastomotic sites from implantation until 11.5 months (0, 2.5, and 11.5 months) identified only graft material without vascularization and mucosal lining, and instead anastomotic inflammation, necrosis and infection (Figure 1, $B, E$, and $H$ ). All CT scans of the chest from 4 weeks until 31.5 months after implantation (4 weeks, 5.5, $27,28,29.5,30,30.5$, and 31.5 months) demonstrated graftassociated pathology including graft exposed to ambient air, anastomotic obstruction, tracheobronchial-esophageal-mediastinal fistulae, airway and esophageal stents, and detached graft from adjacent tissues, as exemplified by Figure 1, $C$, $G$, and $I$ to $K$, and Figures E5, $A$ to $C$, and E9.

\section{Patient 2}

The second patient was a 30-year-old man with a primary adenoid cystic carcinoma of the trachea. The tracheal wall was significantly thickened by the tumor mass without encroachment of the tracheal lumen (Figure 2, A). The patient was admitted to KUH for synthetic trachea implantation. On admission, the patient was asymptomatic. Based on preoperative CT evaluation, a Y-shaped electrospun polyblend PET/PU (polyethylene terephthalate [PET] and polyurethane [PU]; Nanofiber Solutions, Hilliard, Ohio) tracheobronchial graft was prepared using a similar protocol as in patient 1 (Table 1). At surgery (November 2011), the mid- and distal trachea and proximal main bronchi were resected and the BM-MNC-seeded graft implanted followed by administration of the "regenerative boosting therapy." No biopsies were obtained from this graft before implantation to verify cellular lining of the graft in comparison to patient 1 . Postoperative CT scan at 12 days and 2 months demonstrated signs of tracheomediastinal fistula and graft directly exposed to ambient air (Figure 2, $B$ to $D$ ). While still on the "regenerative boosting therapy" multiple central arterial and venous thrombi were diagnosed (Table 2).

The bronchoscopy at 2 months after implantation demonstrated a graft without mucosal lining but covered with a layer of protein precipitate (Figure 2, E and $F$, Video 9, and Figure E12, $A$ to $I$ ). Protein precipitate is a nonliving layer and results from protein leakage into the graft. Protein precipitation does not imply establishment of mucosal lining. The graft biopsies at this time point showed only protein precipitate and graft material without mucosal lining colonized with bacteria and fungi (Figure 2, G). All CT scans of the chest from 12 days until 2 months after implantation (12 and 15 days, and 2 months) demonstrated graft-associated pathology including graft exposed to ambient air and a suspected tracheomediastinal fistula, and suspected granulation tissue at the lower anastomosis, as exemplified by Figure 2, $B$ to $D$. The patient died 

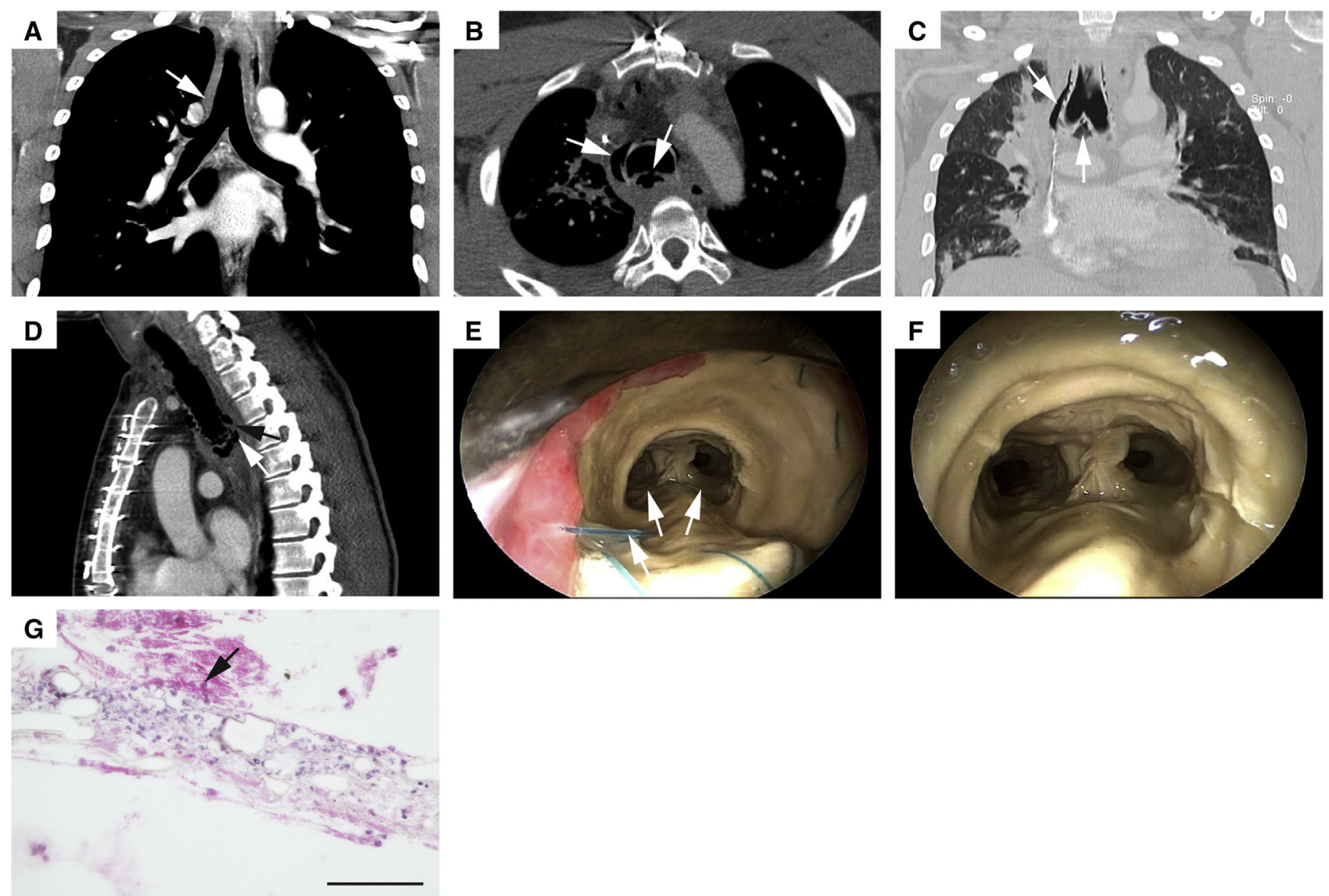

FIGURE 2. Bronchoscopic, radiologic, and histologic findings in patient 2. A, Preoperative computed tomography (CT) scan of the chest 4 weeks before graft implantation. Arrow: tumor mass thickening the tracheal wall between the lower edge of the thyroid gland down to $5 \mathrm{~mm}$ above the right main bronchus departure. No impending tracheal obstruction. B, CT scan of the chest 12 days after graft implantation. Left arrow: graft in direct contact with air outside the graft. Right arrow: suspected tracheomediastinal fistula at the posterior graft wall. C, CT scan of the chest 12 days after graft implantation. Arrows: graft in direct contact with air outside the graft. D, CT scan of the chest 2 months after graft implantation. Upper black arrow: suspected tracheomediastinal fistula region. Lower white arrow: air-filled space beneath the graft. Graft in direct contact with ambient air indicates graft infection and inability of the synthetic graft to integrate into adjacent tissues. E, Bronchoscopy 2 months after graft implantation. Inner graft wall without mucosal lining. Arrows from left to right: exposed anastomotic sutures and the left and right graft bronchi divided by the synthetic graft carina. F, Bronchoscopy 2 months after graft implantation. View advanced in distal direction compared with (E). The inner graft wall without mucosal lining, completely covered by a nonliving layer of yellow-gray-colored protein precipitate (result from protein leakage into the graft and does not imply establishment of mucosal lining). G, Biopsy from the inner graft wall 2 months after graft implantation. Graft material surrounded with protein precipitate with large amounts of inflammatory cells. Mucosal lining could not be identified under the layer of protein precipitate. Gram and Grocott staining verified fungal (black arrow) and bacterial colonization periodic acid-Schiff staining $\times 40$ (scale bar $=50 \mu \mathrm{m})$.

abroad in March 2012, 3.5 months after implantation without the cause of death being disclosed.

\section{Patient 3}

The third patient was a 22-year-old woman who underwent bilateral thoracoscopic sympathectomy abroad for palmar hyperhidrosis in 2011 that was complicated by a right-sided tracheal injury. The lesion started $5 \mathrm{~cm}$ distal to the vocal cords extending down to the right main bronchus. Attempts to repair the injury were without success. The patient developed a chronic right-sided tracheopleural fistula that was drained through a Heimlich valve. The patient was living at home and was monitored for nearly 1 year at the outpatient clinic at the local hospital before admission to KUH for synthetic trachea implantation. A preimplant exploratory thoracotomy was performed in July 2012 where, due to complications, an emergency right-sided pneumonectomy and tracheal resection were performed. In August 2012, 2 weeks after tracheal resection, a tracheal graft made of the same electrospun polyblend PET/PU as patient 2 had received was implanted with the same pre- and postoperative protocol that was used in both previous patients (Table 1). ${ }^{14,16}$ Eighteen days after implantation, a large tracheoesophagealmediastinal fistula was diagnosed, encompassing $25 \%$ of 


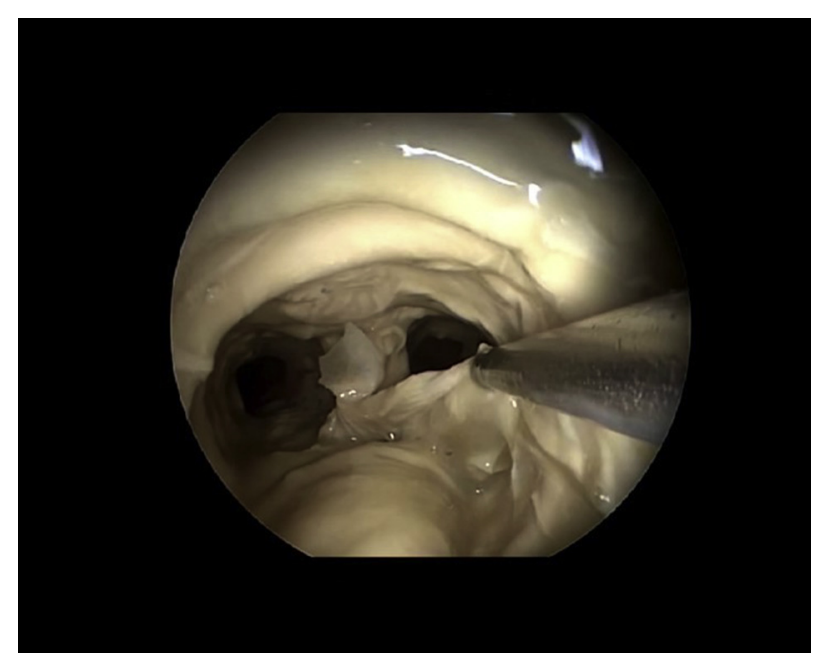

VIDEO 9. Patient 2. Bronchoscopy 2 months after implantation. Video available at: https://www.jtcvs.org/article/S0022-5223(19)32371-2/fulltext.

the distal anastomotic circumference (Figure $3, A$ and $B$, and Figures $\mathrm{E} 13, A$, to E14, $B$ ). This caused a massive air leak between the graft, esophagus, and through the open right-sided thoracotomy, which resulted in the need for graft and esophageal stenting to enable ventilation. The graft biopsy at 3 months after implantation could not identify any mucosal lining but instead bacterial colonization (Figure 3,C). The initial thoracotomy had to be left open to drain accumulating pus from the pneumectomy cavity to prevent spilling over into the airway through the fistulae and remained so until allogeneic tracheal and lung transplantation was performed 3 years and 10 months later (Figure 3,D).

Four months after implantation, the graft itself started to collapse, necessitating stenting of the entire graft and native left bronchus. The patient had to be connected to an airway humidifier at all times, and bronchoscopies were performed through the tracheostomy every fourth hour (6-15 times a day) during the patient's more than 3-year stay in the intensive care unit at KUH (Table 2).

Eleven months after implantation, the situation again became immediately life-threatening, extracorporeal membrane oxygenation was initiated to secure ventilation, and the graft was in July 2013 exchanged for a second graft made of a different synthetic composition (electrospun PET; Harvard Apparatus Regenerative Technology) together with a new course of "regenerative boosting therapy" (Table 1). The reimplantation did not alleviate the tracheoesophageal-pleural fistulae and a transhiatal esophagectomy was performed 1 month after the second graft implantation. One month later, in September 2013, a rightsided thoracoplasty (resection of ribs 2-8) was performed in order to achieve a unilateral chest collapse to prevent the massive air leakage through the anastomotic fistulae (graft exposed to ambient air by both the airway and the open window thoracostomy (pneumectomy cavity)
(Figure 3,D). This procedure was unsuccessful, and the graft never developed a mucosal lining or integrated into adjacent tissues. The related pathology to both the first and second graft including the graft biopsy at 8 months after the second implantation are shown in Figure 3, $A$ to $I$.

The patient suffered from numerous complications including 40 near-fatal suffocation episodes due to graft-associated complications (graft obstruction and collapse, infection, aspiration, bleeding from anastomotic granulation tissue, air leakage through the fistulae, and multiple graft stent dislocations) (Table 2). Fistula leakage and frequent stent dislocations necessitated the development of tailor-made airway stents (Leufen Medical, Berlin, Germany). The patient endured 191 surgical reinterventions, including 57 airway stent interventions and more than 7600 (sic) flexible bronchoscopies. The patient was placed on extracorporeal membrane oxygenation 3 times for a total of 72 days due to an insecure airway and was on ventilator support for a total of 305 days (Table 2 and Table E1). After 37 months in the intensive care unit at $\mathrm{KUH}$, the patient was transferred to a thoracic transplant center for a combined trachea and left lung transplantation, which was performed in May 2016, 2 years and 11 months after implantation of the second synthetic tracheal graft. In March 2017, 9 months after transplantation, the patient died from major airway bleeding without having been discharged from intensive care for 4 years, 7 months, and 24 days.

All bronchoscopies between 6 weeks and 6.5 months after the first graft implantation (6 and 7 weeks, $2,3,3.5,5.5$, and 6.5 months) demonstrated graft-associated complications with anastomotic fistulae and absent mucosal lining as exemplified by the Videos 10 to 12 and Figures E13, A, to E18. The biopsy from the first graft ( 3 months) and second graft and its anastomotic sites between 8 and 23 months after implantation $(8,17,20,21$, and 23 months) could not identify any mucosal lining, only extensive inflammation, granulation tissue, and fibrosis. The biopsy findings from the second graft were supported by all bronchoscopic recordings between 2.5 and 25 months after the second implantation $(2.5,3$, $3.5,4.5,5,6,6.5,8,10,10.5,18$, and 25 months) demonstrating anastomotic fistulae and absent mucosal lining as exemplified by the Videos 13 and 14 and Figures E19, A, to E21. All CT scans of the chest between 15 days and 10.5 months after the first graft implantation (15 and 18 days, 3 weeks, 1, 2.5, 3, 5.5, 9.5, and 10.5 months) and 15 days and 17 months after the second graft implantation (15 days, $1,3,5,8,14.5$, and 17 months) demonstrated graftassociated pathology including graft exposed to ambient air, anastomotic obstruction, graft deformation, tracheomediastinal-pleural-esophageal fistulae, and airway stents, as exemplified by Figures 3, $D$ and $I$, and Figures E15 and E18.

\section{DISCUSSION}

The main finding of this study is that all endoscopic, histologic, and radiologic examinations of these 4 

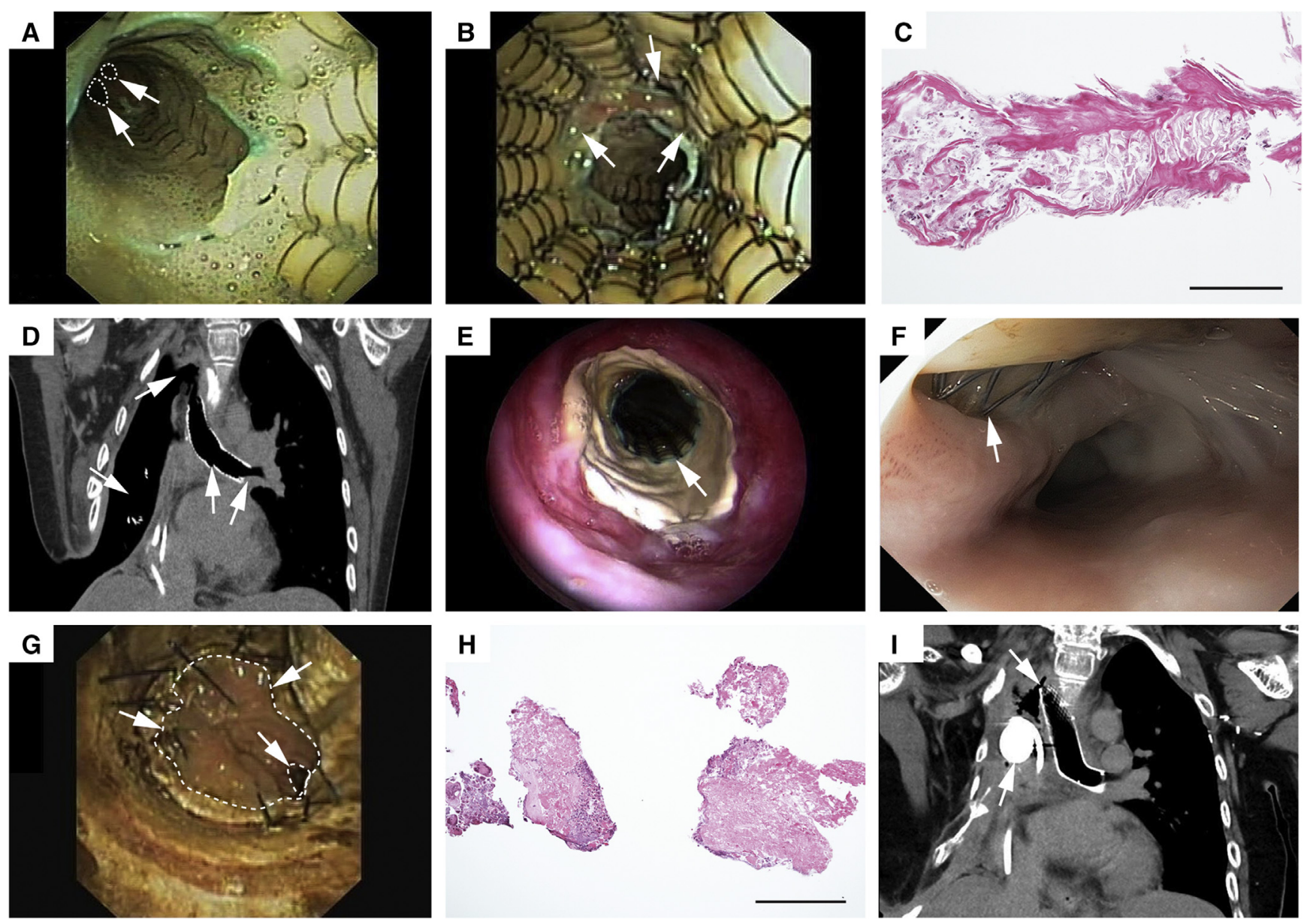

FIGURE 3. Bronchoscopic, histologic, and radiologic findings in patient 3. A, Bronchoscopy 6 weeks after the first graft implantation. Inner graft wall (yellow colored) without mucosal lining seen between the stent wires. Two airway stents cover the entire graft from the proximal graft anastomosis (outside of image) down to the native left upper and lower lobe departures (arrows and dotted circles). Massive amounts of foamy mucus leaking into the airway from the esophagus and pleural cavity by the distal anastomotic fistula (located at the green stent thread marking the distal graft ending). B, Bronchoscopy 2 months after the first graft implantation. Inner graft wall without mucosal lining. Left and right lower arrows: boundaries of distal anastomotic fistula opening extending $25 \%$ of the anastomotic circumference (partly covered by mucus). Upper arrow, visible part of fistula opening. C, Biopsy from the graft's anastomotic region 3 months after the first graft implantation. Mucosal lining could not be identified. Minimal shivers of nonviable cells resembling degenerated squamous epithelial cells and large amounts of bacteria and some inflammatory cells. Hematoxylin and eosin (H\&E) staining $\times 20$ (scale bar $=100 \mu \mathrm{m})$. D, Computed tomography $(\mathrm{CT})$ scan of the chest 3.5 months after the first graft implantation. Arrows from left to right: open window thoracotomy, proximal graft directly exposed to ambient air through the pneumectomy cavity and the airway, distal anastomotic fistula, and obstruction of the proximal part of the native bronchus by scaring and granulation tissue at the distal stent ending (maximal luminal diameter $4.7 \mathrm{~mm}$ ). Graft in contact with ambient and contaminated air indicate graft infection and inability of the graft to integrate into adjacent tissues. E, Bronchoscopy 6.5 months after the first graft implantation. Native proximal trachea and the white-yellow-colored graft without mucosal lining. Arrow: airway stent to obliterate fistula leakage and prevent graft from collapse (proximal graft oval shaped) due to material fatigue. F, Esophagoscopy 6.5 months after the first graft implantation. Arrow: large tracheoesophageal fistula on the anterior esophageal wall with airway stent inserted in the tracheal graft covering the distal graft dehiscence between the graft and the native left main bronchus with an attempt to block leakage from the pleural cavity and the esophagus into the airway. G, Bronchoscopy 3.5 months after the second graft implantation. Inner graft wall without mucosal lining (only covered by desiccated mucus) with the large anastomotic size mismatch between the graft and the native left main bronchus opening, and exposed anastomotic sutures (black straight lines). Large dashed circle: distal graft ending. Small dashed circle with arrow: native left main bronchus opening. Upper left and right arrows: boundaries of the anastomotic fistula at the distal graft ending. H, Biopsy from the graft's anastomotic region 8 months after the second graft implantation. Anastomotic granulation tissue. Mucosal lining could not be identified H\&E staining $\times 10$ (scale bar $=100 \mu \mathrm{m}$ ). I, CT scan of the chest 17 months after the second graft implantation. Upper arrow: tracheopleural fistula at the proximal graft anastomosis with airway stent in the graft. Lower arrow: Fogarty catheter inserted into the open window thoracotomy (pneumectomy cavity) to maintain continuous drainage of accumulating pus. Graft in direct contact with ambient air and pus in the pleural cavity indicate chronic graft infection. 


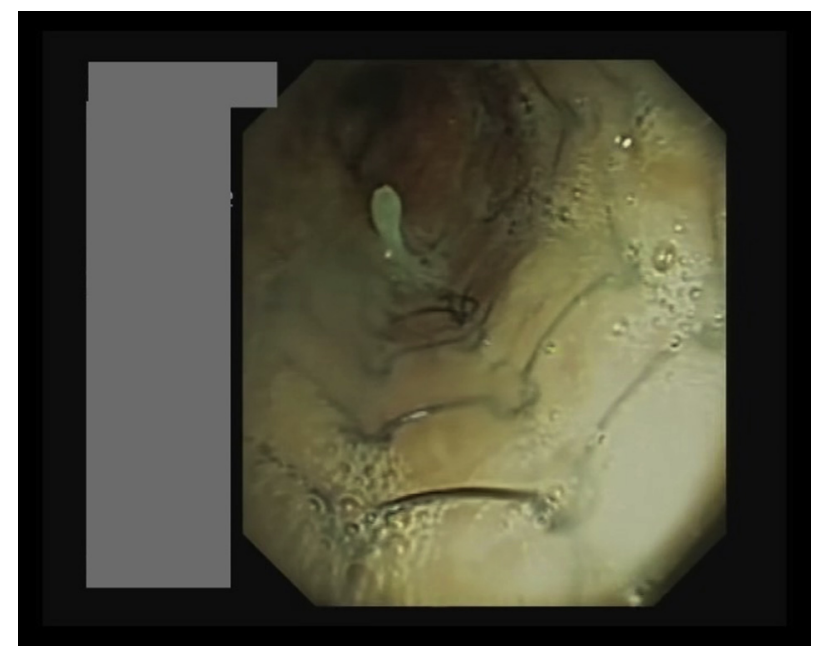

VIDEO 10. Patient 3. Bronchoscopy 6 weeks after the first implantation. Video available at: https://www.jtcvs.org/article/S0022-5223(19)32371-2/ fulltext.

procedures demonstrate that synthetic tracheal grafts seeded with BM-MNCs neither generate vascularization or epithelialization nor become living functional grafts integrated into adjacent tissues. Instead, these grafts caused severe complications and need for multiple reinterventions. This is in stark contrast to the previous reports by Macchiarini and colleagues. ${ }^{14-17}$

The biopsies obtained from the graft immediately before implantation in patient 1 failed to demonstrate any cellular lining. Absence of a confluent cellular layer at implantation is a key finding and indicates that these grafts were unprotected from immediate contamination by ambient air and secretions. Bacterial contamination will inevitably lead to chronic graft infection and anastomotic breakdown., Furthermore, the absence of adhering cells challenges the theory that, by incubating synthetic grafts with

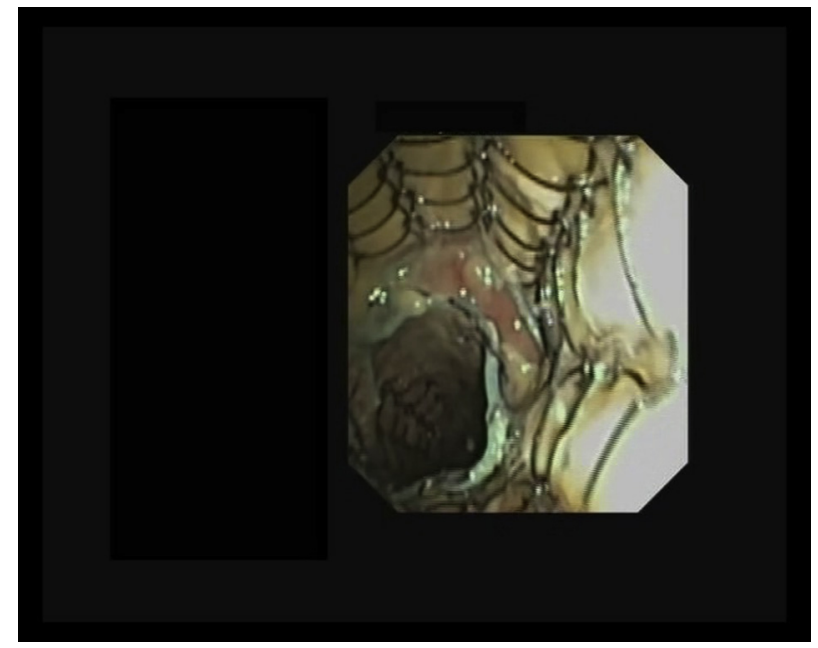

VIDEO 11. Patient 3. Bronchoscopy 2 months after the first implantation. Video available at: https://www.jtcvs.org/article/S0022-5223(19)32371-2/ fulltext.

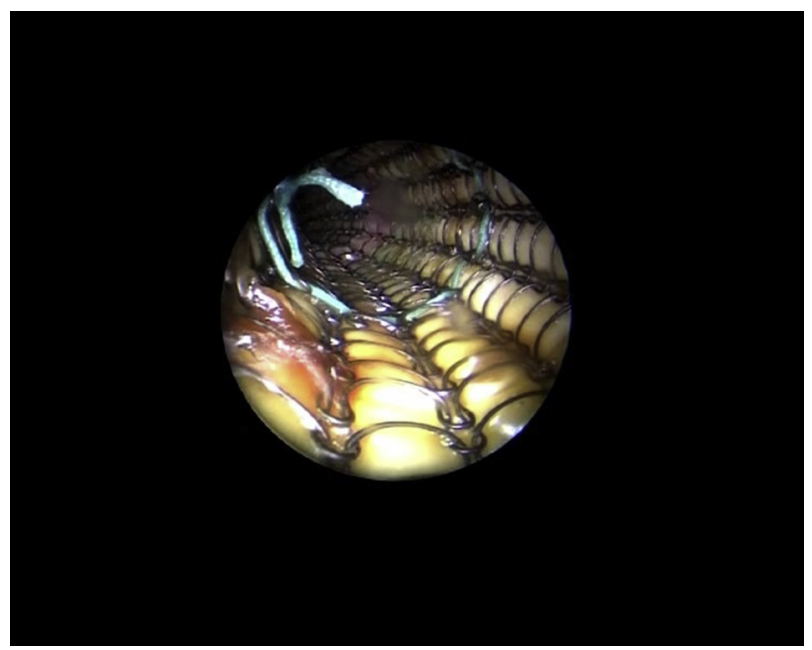

VIDEO 12. Patient 3. Bronchoscopy 6.5 months after the first implantation. Video available at: https://www.jtcvs.org/article/S0022-5223(19) 32371-2/fulltext.

BM-MNCs, these grafts would develop into living functional tracheal tissues with organ-specific epithelialization. ${ }^{14}$ There is no independent scientific foundation to support the theory that either a cellular layer or the fractional stem cell component of a bone marrow aspirate, when applied to a synthetic tube, has the intrinsic ability to generate functional tracheal tissues in situ by itself or by recruitment of progenitor cells. ${ }^{7,14-16,30}$ This applies not only to these procedures after 36 hours of incubation but also in general, irrespective of the administration of the scientifically unfounded "regenerative boosting therapy. ${ }^{, 7,10}$ No animal testing to verify such a transformation of synthetic grafts into living and functional tracheae and their integration into adjacent tissues preceded the initiation of these experiments in humans.

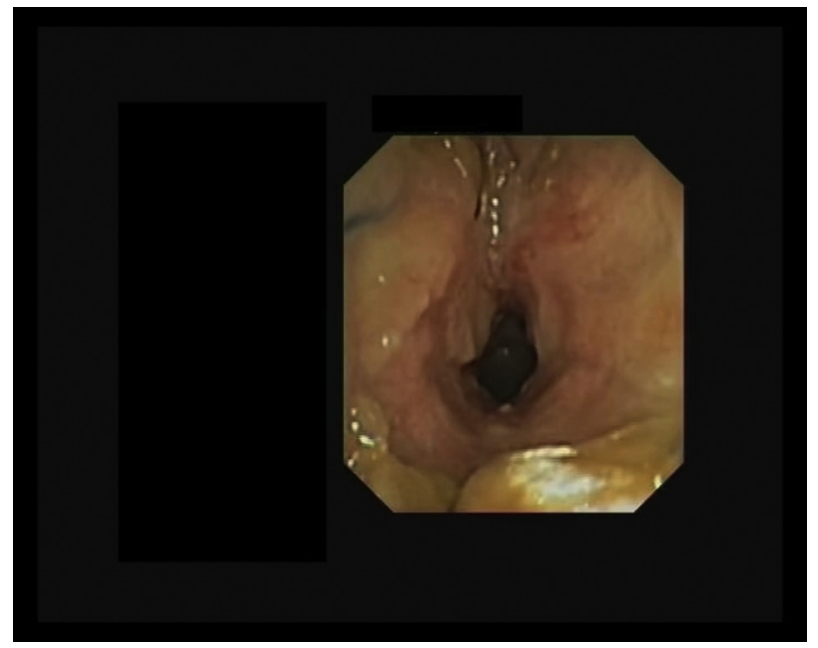

VIDEO 13. Patient 3. Bronchoscopy 3 months after the second implantation. Video available at: https://www.jtcvs.org/article/S0022-5223(19) 32371-2/fulltext. 


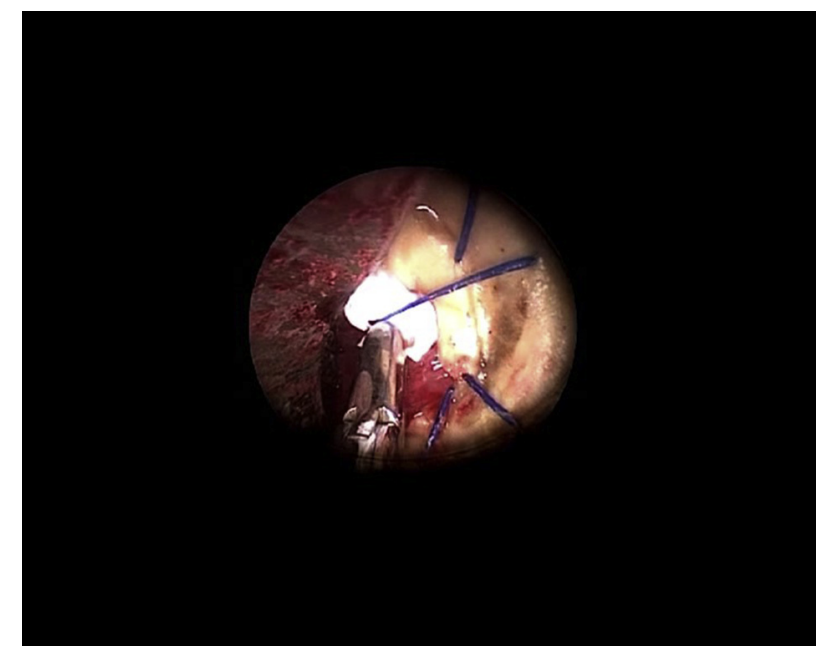

VIDEO 14. Patient 3. Bronchoscopy 6 months after the second implantation. Video available at: https://www.jtcvs.org/article/S0022-5223(19) 32371-2/fulltext.

The absence of cellular lining of the graft implanted in patient 1 suggests that all patients received synthetic grafts that were immediately contaminated. This is supported by all bronchoscopic, CT, and histologic findings shown in this study and confirms what can theoretically be expected after implantation of a synthetic prosthesis in this position. ${ }^{10,31}$ Absence of a preimplant cellular lining equates these 4 procedures to the previous attempts of tracheal replacement with synthetic grafts that were initiated in the second half of the 1940s. ${ }^{8}$

Neither the lack of a cellular layer covering the graft at implantation nor the findings in the first patient at 2.5 months after implantation changed the focus to the fundamental issue of the biological prerequisite of vascularization and mucosal generation but instead led to further exchanges of the synthetic material.

Furthermore, no definite explanation was found in the medical records on the indication to subject patient 1 to experimental tracheal replacement, specifically considering the tumor size (Table 1) evident on preoperative bronchoscopy, CT, and also later supported by the postoperative pathological examination. Not only because there was no previous in vivo data to support the hypothesis that normal tracheal function could be restored with a synthetic tube incubated with growth factors and BM-MNCs but also because other reconstructive procedures potentially could have been attempted.

All patients developed major thromboembolic events while on the "regenerative boosting therapy" despite the fact that none of the patients had a known coagulative disorder and all 3 patients being on conventional postoperative thrombosis prophylaxis. It cannot be ruled out that these complications were directly related to the administration of off-label use of epoetin in supratherapeutic doses, ie, 40,000 UI every other day for 12 to 16 days (Table 1). ${ }^{14}$ Regarding the use of
TGF- $\beta 3$ for graft conditioning immediately before graft implantation, we were not able to further analyze the individual risk to which these patients were subjected beyond noting that TGF- $\beta 3$ is a laboratory substance neither approved for human nor animal use due to the risk of viral transfer between species. ${ }^{32,33}$

\section{CONCLUSIONS}

This is the first long-term follow-up study of synthetic tracheal grafts seeded with BM-MNCs implanted in humans. All findings demonstrate that these grafts were not cellularized with site-specific cells and did not become living and functional grafts that integrated into adjacent tissues. These procedures were associated with severe complications directly associated to the grafts and caused the patients significant suffering and death.

\section{Conflict of Interest Statement}

Authors have nothing to disclose with regard to commercial support.

\section{References}

1. Udelsman B, Mathisen DJ, Ott HC. A reassessment of tracheal substitutes-a systematic review. Ann Cardiothorac Surg. 2018;7:175-82.

2. Minnich DJ, Mathisen DJ. Anatomy of the trachea, carina, and bronchi. Thorac Surg Clin. 2007; 17:571-85.

3. Furlow PW, Mathisen DJ. Surgical anatomy of the trachea. Ann Cardiothorac Surg. 2018;7:255-60.

4. Delaere P, Lerut T, Van Raemdonck D. Tracheal Transplantation: state of the art and key role of blood supply in its success. Thorac Surg Clin. 2018;28:337-45.

5. Salassa JR, Pearson BW, Payne WS. Gross and microscopical blood supply of the trachea. Ann Thorac Surg. 1977;24:100-7.

6. Grillo HC. Tracheal replacement: a critical review. Ann Thorac Surg. 2002;73: 1995-2004.

7. Delaere P, Van Raemdonck D. Tracheal replacement. J Thorac Dis. 2016;8:S186-96.

8. Virk JS, Zhang H, Nouraei R, Sandhu G. Prosthetic reconstruction of the trachea: a historical perspective. World J Clin Cases. 2017;5:128-33.

9. Den Hondt M, Vranckx JJ. Reconstruction of defects of the trachea. J Mater Sci Mater Med. 2017;28:24

10. Etienne H, Fabre D, Gomez Caro A, Kolb F, Mussot S, Mercier O, et al. Tracheal replacement. Eur Respir J. 2018;51.

11. Deslauriers J, Aubree N, Shamji FM. Experiences with prosthetic airway replacement. Thorac Surg Clin. 2018;28:377-84.

12. Macchiarini P. Trachea-guided generation: deja vu all over again? J Thorac Cardiovasc Surg. 2004;128:14-6.

13. Delaere PR. Tracheal transplantation. Curr Opin Pulm Med. 2012;18:313-20.

14. Jungebluth P, Alici E, Baiguera S, Blomberg P, Bozóky B, Crowley C, et al. Tracheobronchial transplantation with a stem-cell-seeded bioartificial nanocomposite: a proof-of-concept study. Lancet. 2011;378:1997-2004.

15. Badylak SF, Weiss DJ, Caplan A, Macchiarini P. Engineered whole organs and complex tissues. Lancet. 2012;379:943-52.

16. Jungebluth P, Haag JC, Lim ML, Lemon G, Sjöqvist S, Gustafsson Y, et al. Verification of cell viability in bioengineered tissues and organs before clinical transplantation. Biomaterials. 2013;34:4057-67.

17. Del Gaudio C, Baiguera S, Ajalloueian F, Bianco A, Macchiarini P. Are synthetic scaffolds suitable for the development of clinical tissue-engineered tubular organs? J Biomed Mater Res A. 2014;102:2427-47.

18. Delaere PR, Van Raemdonck D. The trachea: the first tissue-engineered organ? J Thorac Cardiovasc Surg. 2014;147:1128-32.

19. Claesson-Welsh L, Hansson GK. Tracheobronchial transplantation: the Royal Swedish Academy of Sciences' concerns. Lancet. 2016;387:942.

20. Vogel G. Regenerative medicine. Report finds misconduct by surgeon. Science. 2015;348:954-5. 
21. The Lancet. The final verdict on Paolo Macchiarini: guilty of misconduct. Lancet. 2018;392:2.

22. The Lancet E. Retraction-Tracheobronchial transplantation with a stem-cellseeded bioartificial nanocomposite: a proof-of-concept study. Lancet. 2018;392:11.

23. Erratum. Retracted: Airway transplantation. Thorac Surg Clin. 2018;28. xi.

24. Gerdin B. Investigation of scientific misconduct. Statement of opinion on assignment ref: 2-2184/2014. 2015. Available at: http://www.circare.org/info/ pm/gerdin-finalrpt-20150513.pdf. Accessed December 7, 2016.

25. Expert Group on Misconduct in Research. The Ethics Review Appeals Board in Sweden. Statement Ref no. O 12-20162017. Available at: https://drive.google. com/file/d/0By2HqPi4t2RbYzZweVRieVVMajhJQUM0cmFMekwyRVJTUFVr/ view. Accessed October 20, 2017.

26. Ajalloueian F, Lim ML, Lemon G, Haag JC, Gustafsson Y, Sjöqvist S, et al, Retraction notice to: "Biomechanical and biocompatibility characteristics of electrospun polymeric tracheal scaffolds" [BIOMATERIALS 35/20 (2014) 5307-5315]. Biomaterials. 2019;199:89.

27. Jungebluth P, Haag JC, Lim ML, Lemon G, Sjöqvist S, Gustafsson Y, et al, Retraction notice to: "Verification of cell viability in bioengineered tissues and organs before clinical transplantation" [BIOMATERIALS (2013) 4057-4067]. Biomaterials. 2019;199:88.
28. The Lancet Editors. Retraction-Engineered whole organs and complex tissues Lancet. 2018:392:11.

29. Sigurdsson MI, Sigurdsson H, Hreinsson K, Simonardottir L, Gudbjartsson T. Bronchiovenous fistula causing bleeding and air embolism: an unusual complication of bronchoscopic tumor resection. Am J Respir Crit Care Med. 2011;183:681-2.

30. Jungebluth P, Macchiarini P. Airway transplantation. Thorac Surg Clin. 2014;24: 97-106.

31. Zimmerli W, Sendi P. Pathogenesis of implant-associated infection: the role of the host. Semin Immunopathol. 2011;33:295-306.

32. Ma H, Galvin TA, Glasner DR, Shaheduzzaman S, Khan AS. Identification of a novel rhabdovirus in Spodoptera frugiperda cell lines. J Virol. 2014;88: 6576-85.

33. Geisler C, Jarvis DL. Rhabdovirus-like endogenous viral elements in the genome of Spodoptera frugiperda insect cells are actively transcribed: implications for adventitious virus detection. Biologicals. 2016;44:219-25.

Key Words: tracheal replacement, tracheal prosthesis, synthetic tracheal graft, airway surgery
See Article page 2525.

\section{Commentary: The sobering truth about tracheal regeneration}

Pierre R. Delaere, MD, PhD, ${ }^{\mathrm{a}}$ and

Dirk Van Raemdonck, $\mathrm{MD}, \mathrm{PhD}^{\mathrm{b}}$

Tracheal replacement to restore functional integrity of a long segmental defect or a complex airway stenosis remains a surgical challenge. ${ }^{1}$ A variety of surgical techniques have been reported clinically, including transplantation of autologous $^{2}$ or heterologous ${ }^{3}$ tissue flaps, direct ${ }^{4,5}$ or indirect $^{6}$ cadaveric tracheal allografting, and interposition of a "regenerated" trachea.

In this issue of the Journal, Fux and colleagues ${ }^{8}$ from the Karolinska Institute in Stockholm publish a very detailed and well-illustrated long-term follow-up of 3 patients who received a "bioengineered tracheal allograft seeded with bone marrow cells" resulting in numerous complications and finally fatal outcome. These authors from Karolinska institute should be congratulated for their courage.

\footnotetext{
From the Departments of ${ }^{\mathrm{a} O t o-R h i n o-L a r y n g o l o g y}$ and ${ }^{\mathrm{b}}$ Thoracic Surgery, University Hospitals Leuven, Leuven, Belgium.

Disclosures: Authors have nothing to disclose with regard to commercial support.

Received for publication Oct 27, 2019; revisions received Oct 27, 2019; accepted for publication Oct 28, 2019; available ahead of print Dec 4, 2019.
}

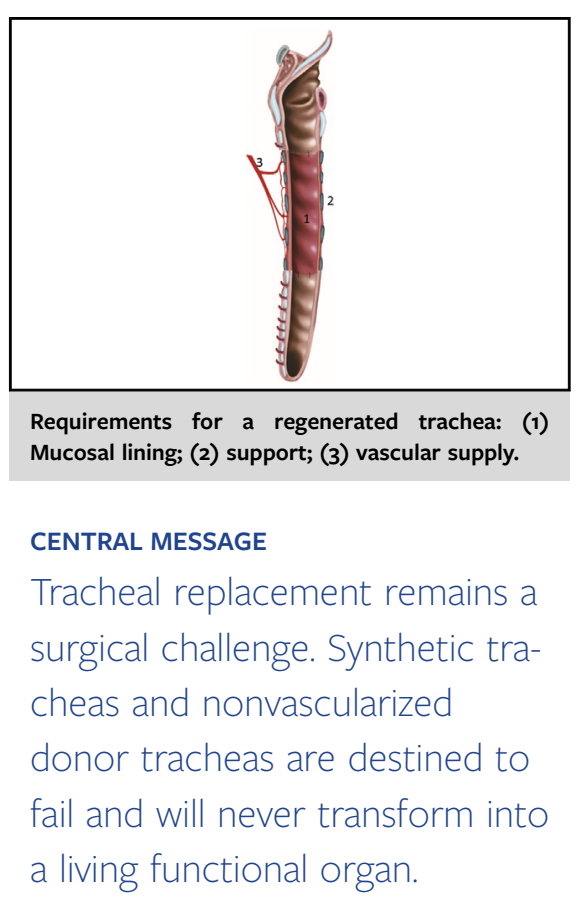

Address for reprints: Dirk Van Raemdonck, MD, PhD, Department of Thoracic Surgery, University Hospital Gasthuisberg, Herestraat 49, B-3000 Leuven, Belgium (E-mail: dirk.vanraemdonck@uzleuven.be).

J Thorac Cardiovasc Surg 2020;159:2537-9 0022-5223/\$36.00

Copyright (C) 2019 by The American Association for Thoracic Surgery

https://doi.org/10.1016/j.jtcvs.2019.10.116 


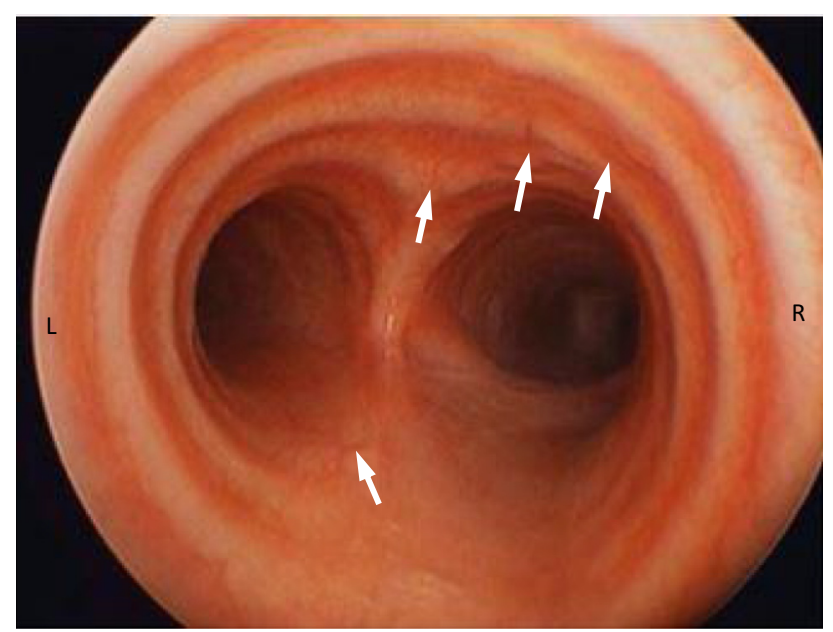

FIGURE E1. Normal human trachea with carina and left and right main bronchus departures. Arrows: vessels demonstrating normal vascularization of the tracheal wall. From Bellamkonda-Athmaram V, Sulman CG, Basel DG, Southern J, Konduri GG, Basir MA. Alveolar capillary dysplasia with multiple congenital anomalies and bronchoscopic airway abnormalities. J Perinatol. 2014;34:326-8. Reprinted with permission from Springer Nature. 

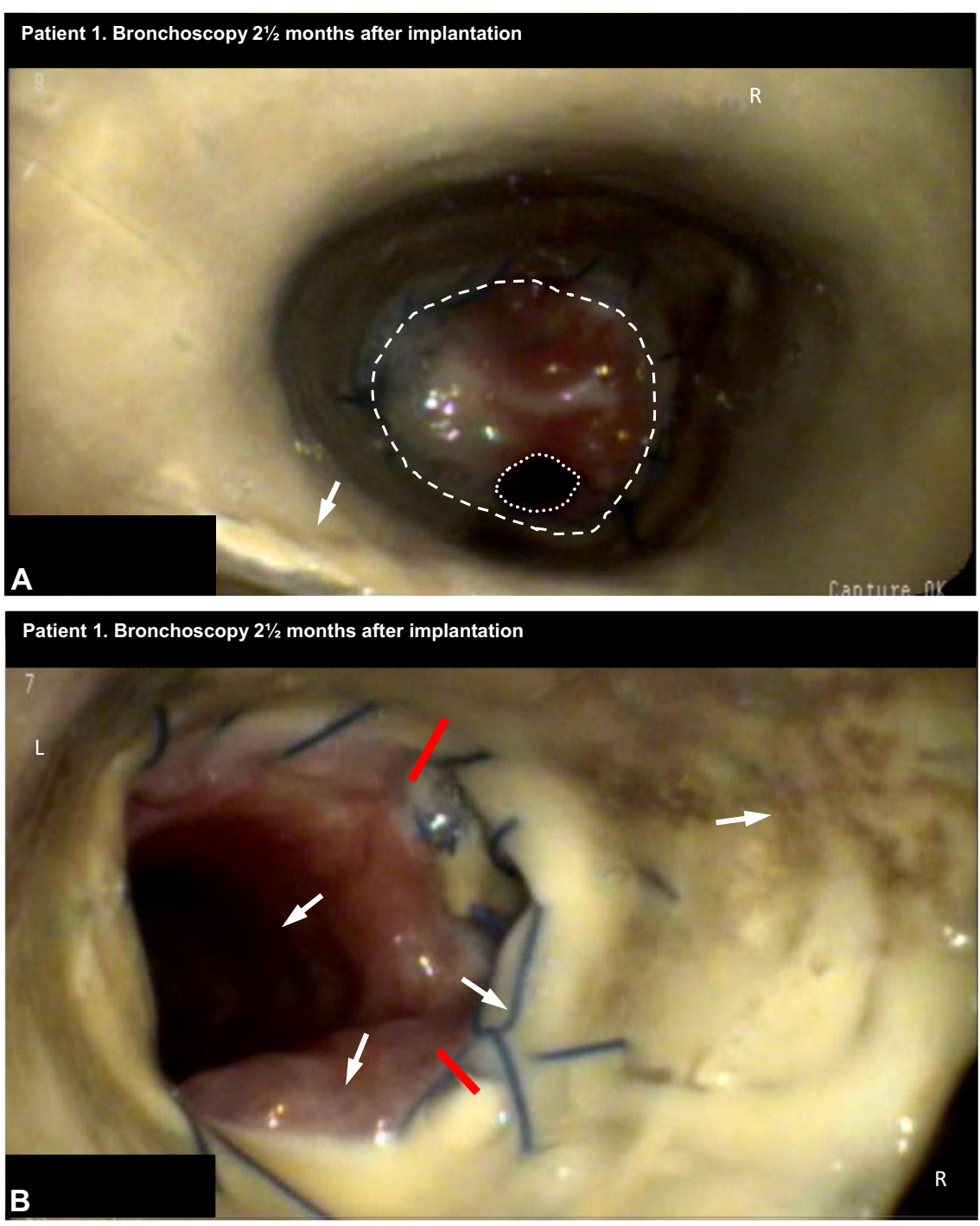

FIGURE E2. Patient 1. Bronchoscopy 2.5 months after implantation of the synthetic tracheal graft. A, Synthetic graft carina with the right distal graft anastomosis. The inner graft wall without vascularization and mucosal lining. Arrow: graft carina. Dashed line: outlines the right distal graft anastomosis. Dotted line: obstructed native right main bronchus opening (expected to have the same area as the dashed line) blocking the right lung from ventilation due to the excessive formation of anastomotic granulation tissue. B, Left distal graft anastomosis. The left distal graft departure without mucosal lining. Arrows from left to right: native left main bronchus opening, granulation tissue, exposed sutures, and brown-colored dispositions of desiccated airway mucus. Red lines: boundaries of the anastomotic fistula extending $25 \%$ of the circumference. 

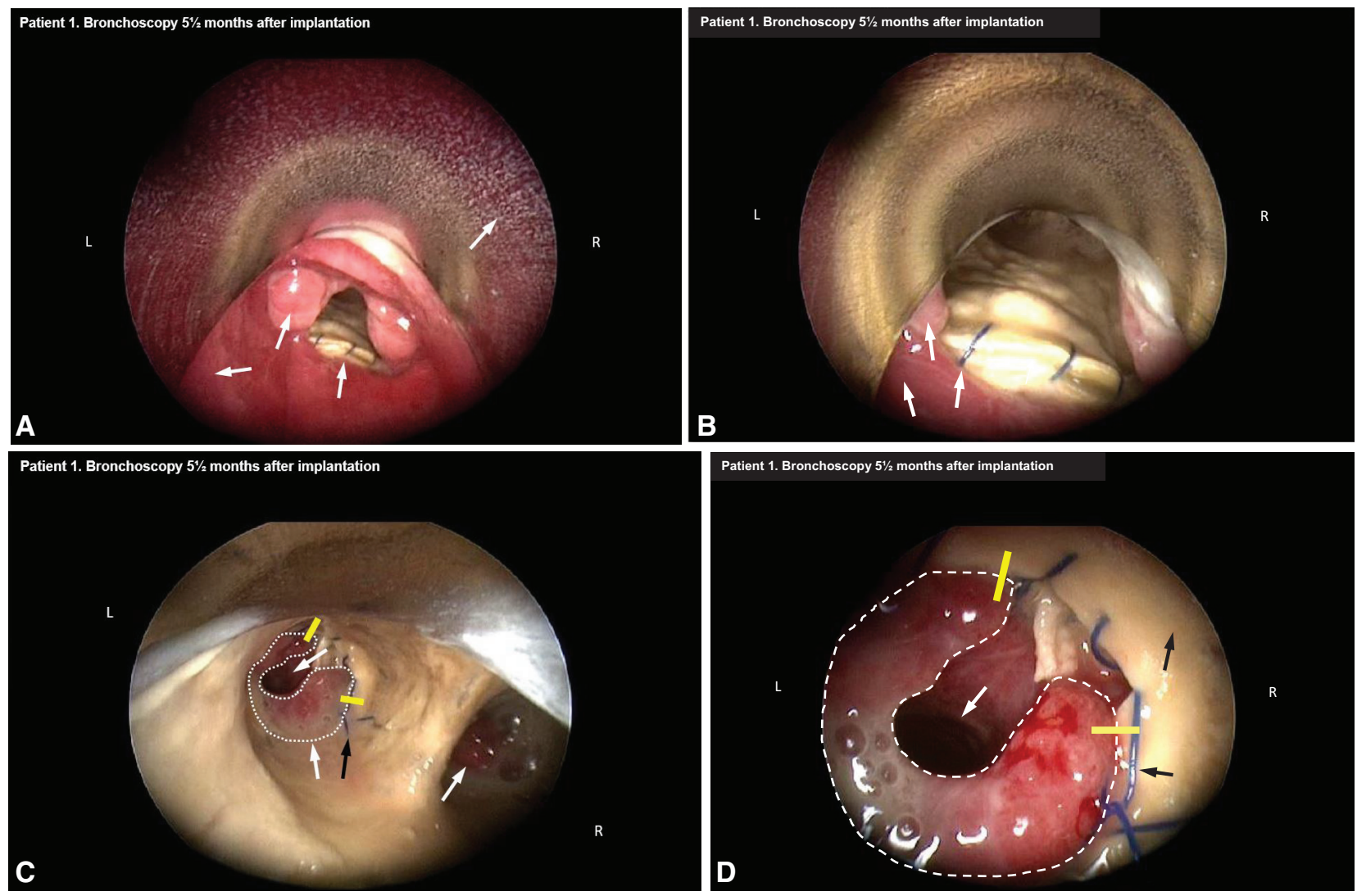

FIGURE E3. Patient 1. Bronchoscopy 5.5 months after implantation of the synthetic tracheal graft. A, Native trachea and the proximal graft anastomosis. View from the native proximal trachea toward the yellow-white-colored graft. Arrows from left to right: native tracheal tissue, obstructing anastomotic granulations, exposed sutures at the proximal graft anastomosis, and the inner wall of the bronchoscope. B, Proximal graft anastomosis and the synthetic graft. View advanced in distal direction. Absence of mucosal lining on the inner graft wall 5.5 months after implantation. Arrows from left to right: native tracheal tissue, anastomotic granulation tissue, and exposed sutures. C, Synthetic graft with obstruction of the left distal anastomosis. View further advanced in distal direction toward the synthetic carina. Inner graft wall without vascularization and mucosal lining. Dashed line: outlines the area of excessive formation of granulation tissue obstructing the left main bronchus opening. Yellow lines: boundaries of the anastomotic fistula extending $25 \%$ of the circumference. Arrows from left to right: obstructed left main bronchus opening, exposed sutures, and the right distal graft departure with complete obstruction of the native right main bronchus opening. Compare with Figure 1 showing a normal viable human trachea. D, Left distal graft anastomosis. View advanced in distal direction towards the left distal graft anastomosis. Dashed line: outlines the area of excessive formation of granulation tissue severely obstructing the left main bronchus. Arrows from left to right: left main bronchus opening, exposed sutures, and the inner graft wall without mucosal lining. Yellow lines: boundaries of the anastomotic fistula extending $25 \%$ of the circumference. E, Right distal graft anastomosis. Left arrow: granulation tissue completely obstructing the right main bronchus opening blocking the right lung from being ventilated. Right arrow: inner graft wall without mucosal lining. F, Right distal graft anastomosis. View advanced in distal direction. The right graft departure toward the distal graft anastomosis. The native right main bronchus opening completely obstructed by granulation tissue. Left arrow: inner graft wall without mucosal lining. Right arrow: exposed and loose anastomotic sutures. G, Right distal graft anastomosis. View advanced in distal direction. Dashed line: outlines the native right bronchus opening being completely obstructed by excessive formation of granulation tissue. Arrows from left to right: bronchoscopic instrument (searching for bronchus opening), inner graft wall without mucosal lining, and loose anastomotic sutures. In the corresponding bronchoscopic video (Video 2) evident that the distal graft ending has not integrated with the native bronchus but instead being detached when manipulated by the bronchoscope. H, Right distal graft anastomosis after resection of granulation tissue. Dashed line: outlines the right distal graft ending. Dotted line: outlines the minor opening established to the right main bronchus by resection of obstructing granulation tissue. 

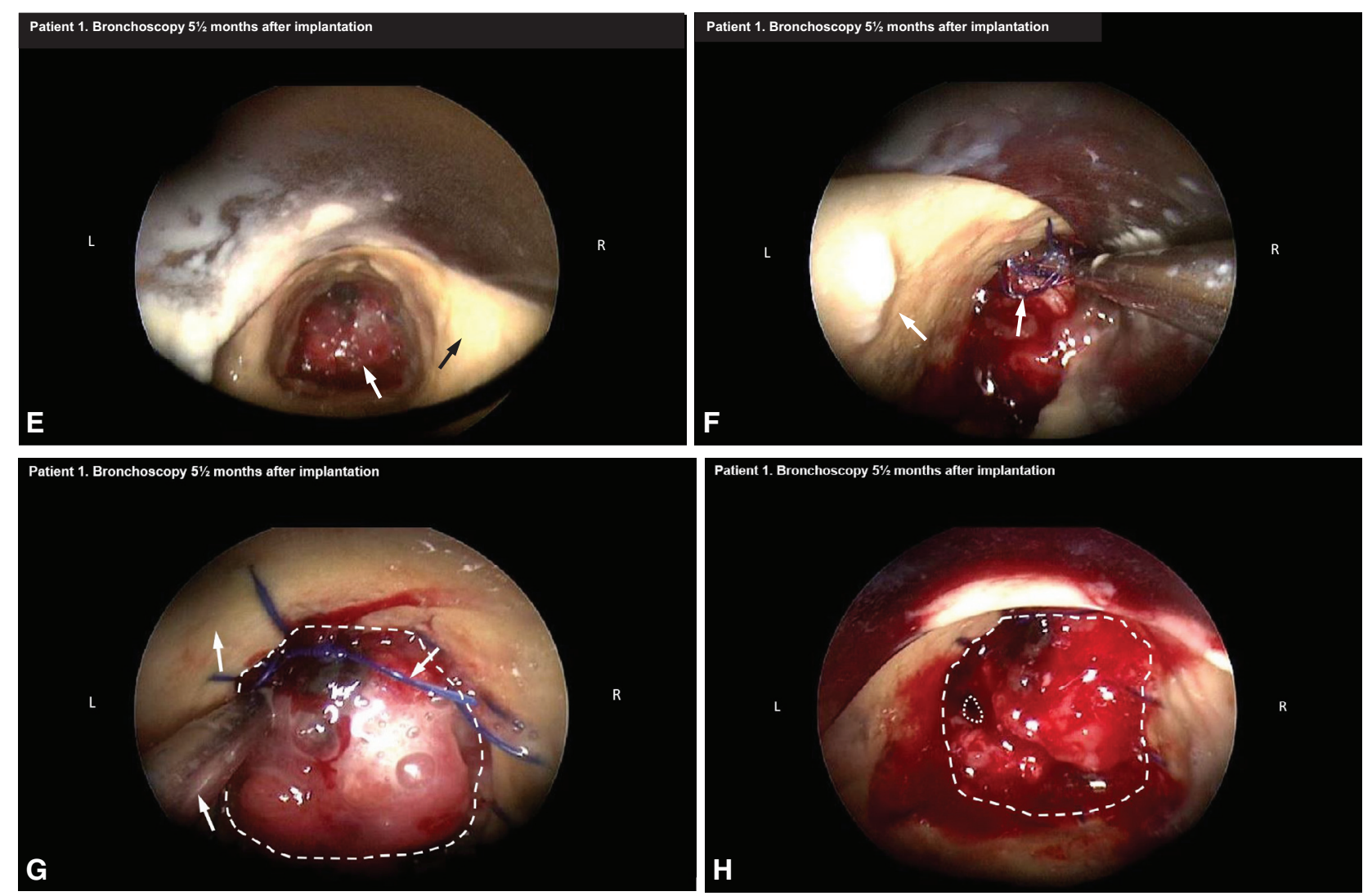

FIGURE E3. Continued 

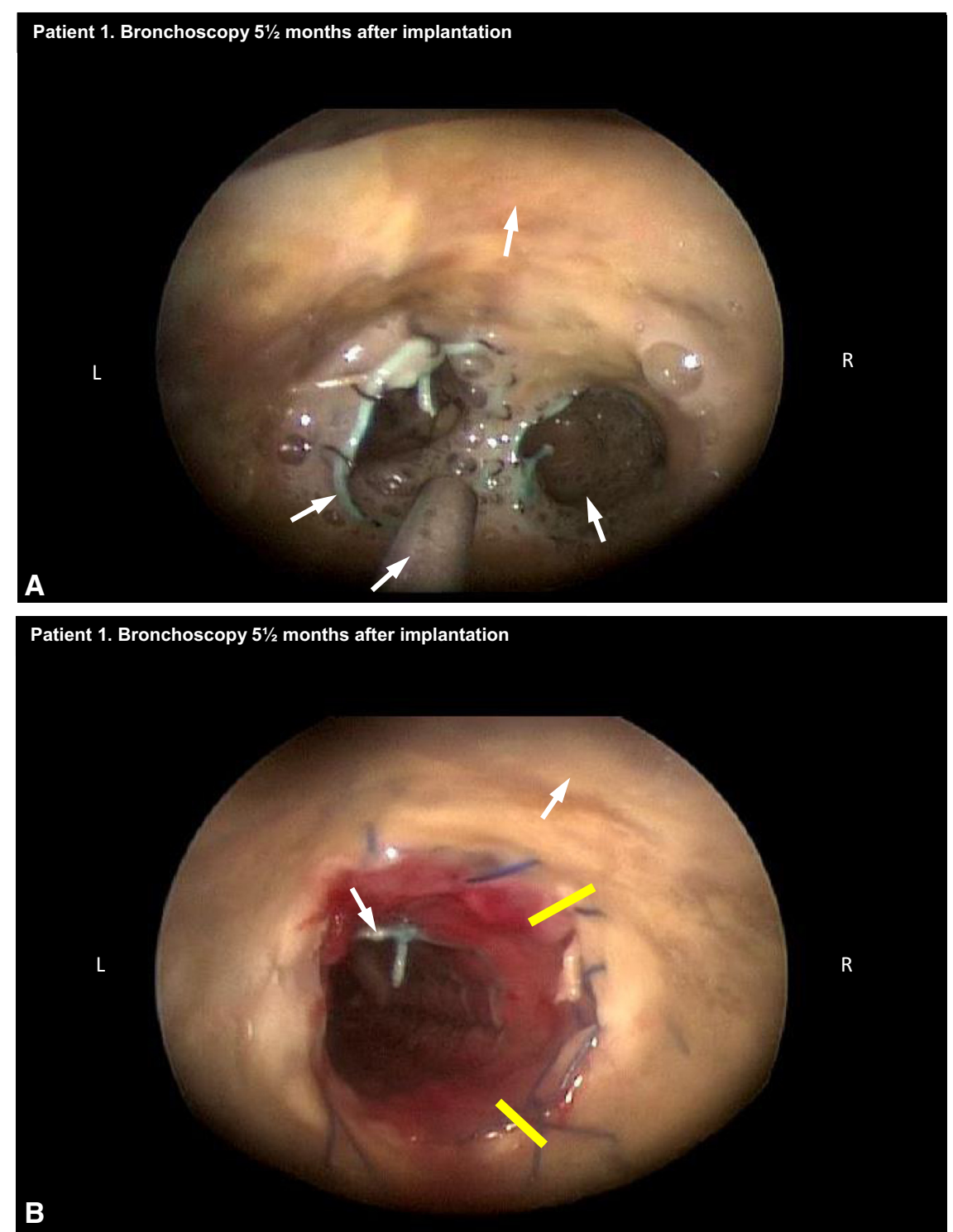

FIGURE E4. Patient 1. Bronchoscopy 1 day later (5.5 months after implantation of the synthetic tracheal graft). A, Synthetic graft with inserted airway stents in the left and right distal graft departures. Arrows from left to right: airway stents in both distal graft departures with an (failed) attempt to keep the main bronchus anastomoses open and to obliterate the fistulae (green threads marking the proximal stent edge), bronchoscopic suction port, inner wall of the graft without mucosal lining, and granulation tissue obstructing the right bronchus opening. B, Left distal graft anastomosis. View advanced in distal direction towards the left distal graft anastomosis. Left arrow: airway stent in the native left main bronchus to attempt to seal the anastomotic fistulae. Right arrow: inner graft wall without mucosal lining. Yellow lines: boundaries of anastomotic fistula extending $25 \%$ of the anastomotic circumference. 

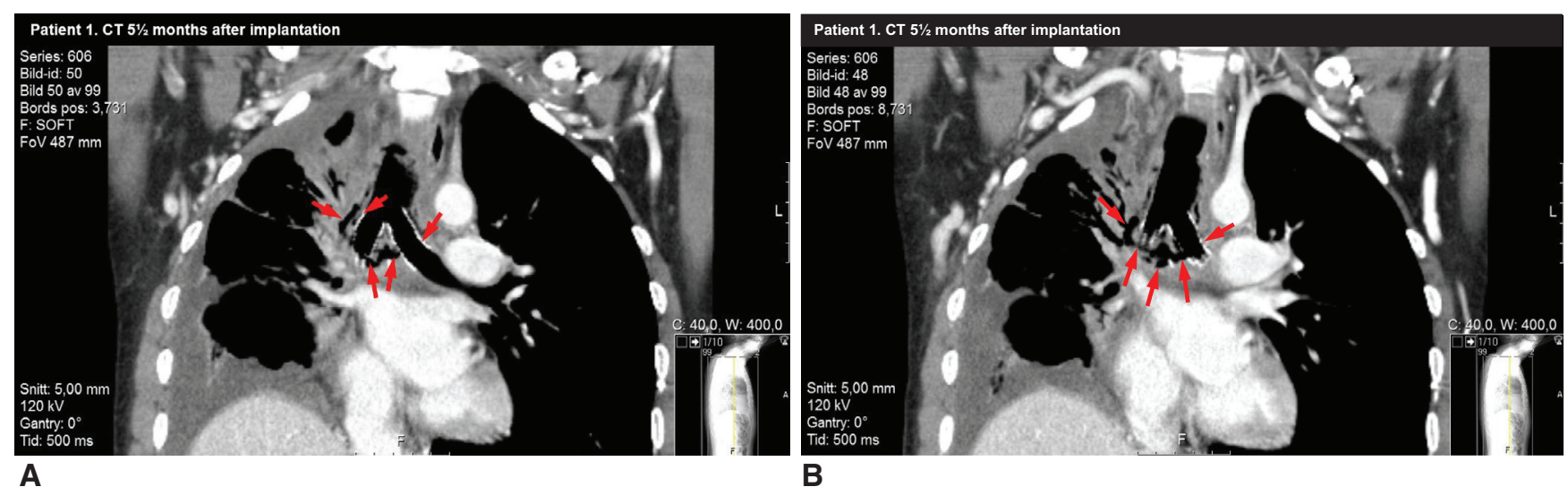

A

B

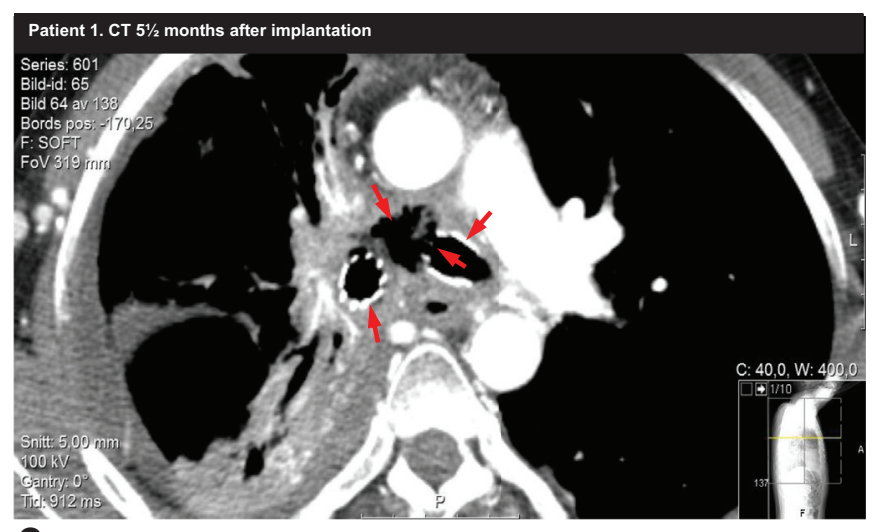

C

FIGURE E5. Patient 1. Computed tomography (CT) 5.5 months after implantation of the synthetic tracheal graft. A, CT of the chest. Synthetic graft with direct air contact. Arrows from left to right: air outside the graft, stent in the right main bronchus, fistula at the right and left distal graft anastomoses, and stent in the left graft departure and left main bronchus. Airway stents have been placed in an (failed) attempt to obliterate the fistulae. The fistulae and air outside the graft are indicative of graft infection and inability of the graft to integrate with the native bronchus and the adjacent tissues. B, CT of the chest. Bilateral distal anastomotic fistulae. Arrows from left to right: airway stent in the right main bronchus, circumferential fistula at the right distal graft anastomosis (the right graft end completely detached from the native right bronchus), air outside the graft, fistula at the left distal graft anastomosis, and airway stent in the left graft departure and left main bronchus. C, CT of the chest. Tracheobronchial-mediastinal fistula. Arrows from left to right: airway stent in the right main bronchus, air outside the graft, large mediastinal fistula at the left distal graft anastomosis, and airway stent in the left main bronchus. 

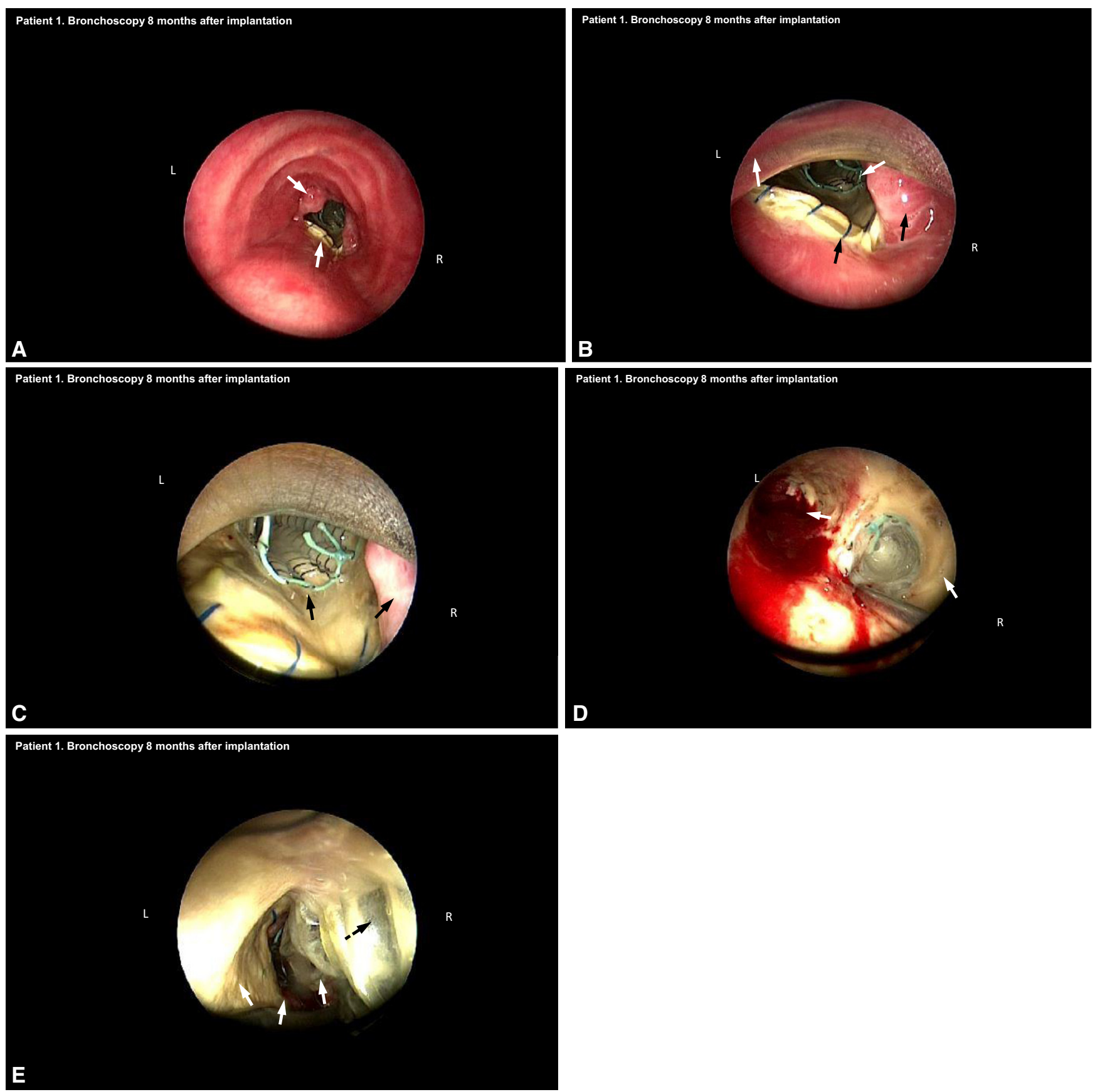

FIGURE E6. Patient 1. Bronchoscopy 8 months after implantation of the synthetic tracheal graft. A, Proximal part of the native trachea. View from the proximal part of the native trachea down towards the upper edge of the graft. Note the normal vascularization of the native tracheal wall. Arrows from left to right: granulation tissue at the proximal graft anastomosis. B, Proximal graft anastomosis. Arrows from left to right: inner wall of the bronchoscope, upper edge of the graft with exposed anastomotic sutures, absence of mucosal lining, airway stent (green thread marking the proximal stent edge), and obstructing anastomotic granulation tissue. C, Proximal part of the synthetic graft. Proximal view on the posterior inner graft wall without vascularization or mucosal lining. Left arrow: dislocated (upwards) airway stent. Right arrow: granulation tissue at the proximal graft anastomosis. D, Synthetic graft with carina, left and right graft departures. View advanced into the graft without mucosal lining. Blood in the left main bronchus opening (originating from extensive resection of obstructing left distal anastomotic granulation tissue). Left arrow, left main bronchus opening. Right arrow, proximal ending of the airway stent (green-colored thread) in the obstructed, and the fluid filled right main bronchus (blocking the right lung from being ventilated). E, Left inner wall of the synthetic graft. Arrows from left to right: left inner graft wall without mucosal lining, left distal anastomosis between graft and the native left main bronchus opening, and the outside of the airway stent which is about to be pulled out and replaced. Dashed arrow: direction of the airway stent being pulled out. 

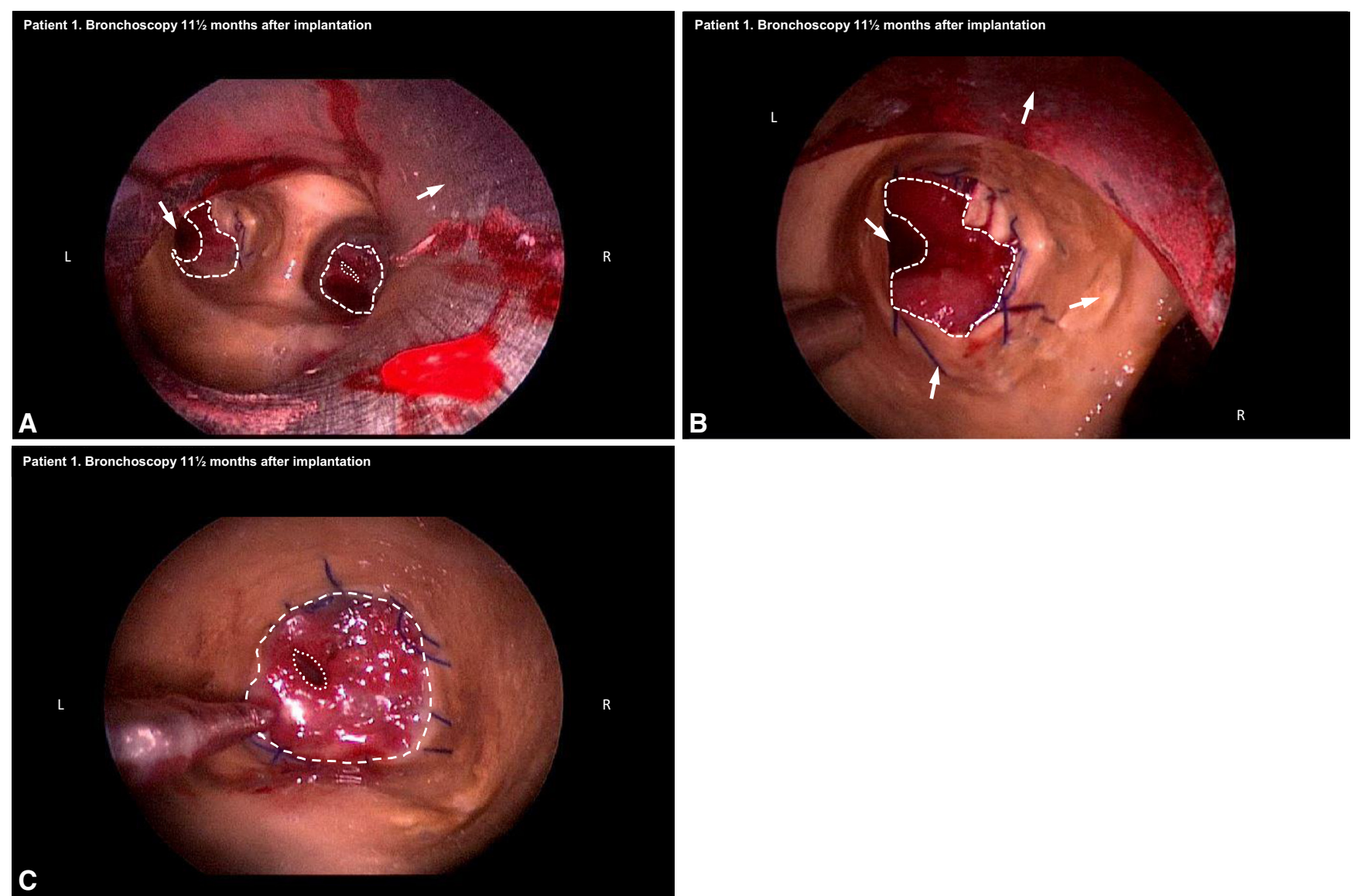

FIGURE E7. Patient 1. Bronchoscopy 11.5 months after implantation of the synthetic tracheal graft. A, Synthetic graft with carina and the left and right distal graft anastomoses. Left and right dashed lines: outlines the areas of granulation tissue obstructing the left and right main bronchus openings. Dotted line: outlines the obstructed opening to the right main bronchus (expected to have the same area as the surrounding dashed line). Left arrow: obstructed opening to the left main bronchus. Right arrow: inner wall of the bronchoscope. B, Left distal graft anastomosis. View advanced in distal direction towards the left distal anastomosis. Dashed line: outlines the area of granulation tissue obstructing the bronchus opening. Arrows from left to right: remaining left main bronchus opening, exposed anastomotic sutures, inner wall of bronchoscope, and graft carina without mucosal lining. C, Right distal graft anastomosis. Absence of mucosal lining on the graft 11.5 months after implantation. Dashed line: outlines the distal graft edge. Dotted line: outlines the obstructed right main bronchus opening (expected to have the same area as the surrounding dashed line). 

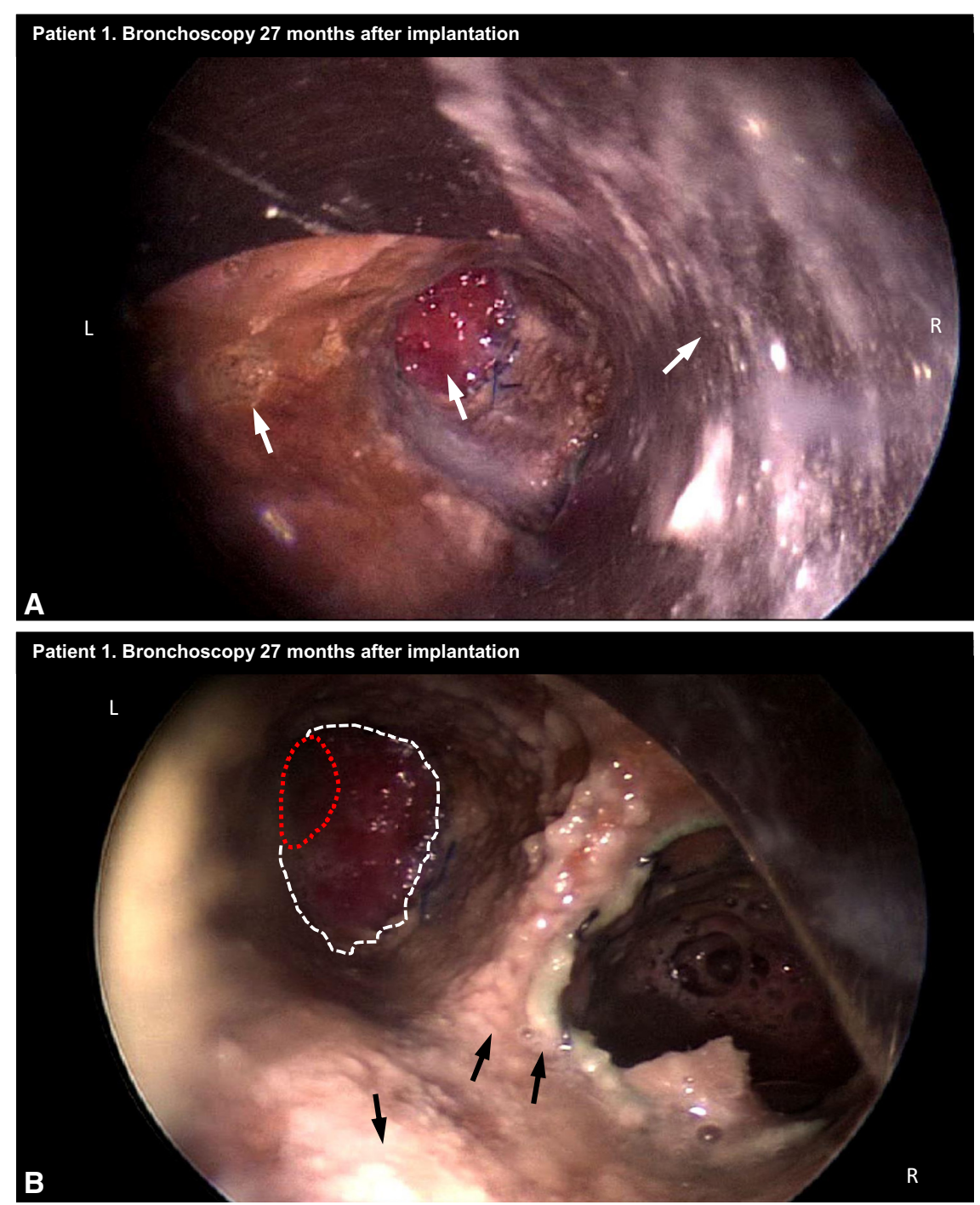

FIGURE E8. Patient 1. Bronchoscopy 27 months after implantation of the synthetic tracheal graft. A, Left and posterior inner graft wall. Arrows from left to right: remaining wall crater from the biopsy 16 months earlier, obstructed left main bronchus opening, and the inner wall of the bronchoscope. Remaining biopsy crater is indicative of absent mucosal lining 27 months after implantation. B, Synthetic graft with obstructed left and right distal graft anastomoses. View advanced in distal direction towards the carina. Bilateral obstruction of distal graft anastomoses due to excessive formation of anastomotic granulation tissue. Dashed line: outlines the area of granulation tissue obstructing the left bronchus opening. Red dotted line: outlines the obstructed left bronchus opening (expected to have the same area as the surrounding dashed line). Arrows from left to right: inner graft wall without mucosal lining, graft carina, and the proximal ending of the airway stent (green-colored thread) in the right distal graft departure. 


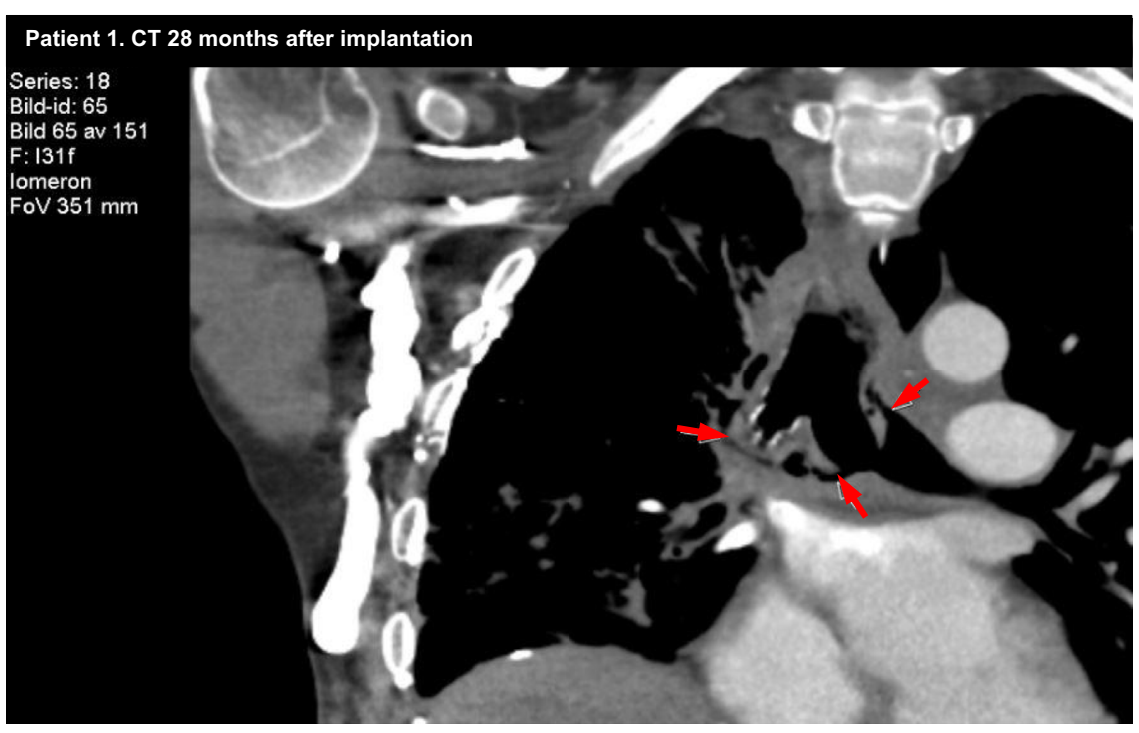

FIGURE E9. Patient 1. Computed tomography (CT) 28 months after implantation of the synthetic tracheal graft. CT of the chest. Synthetic graft detached from the adjacent tissues. Arrow from left to right: left and right distal graft detachment, and air outside the graft by the complete graft detachment from the adjacent tissues and native bronchi.

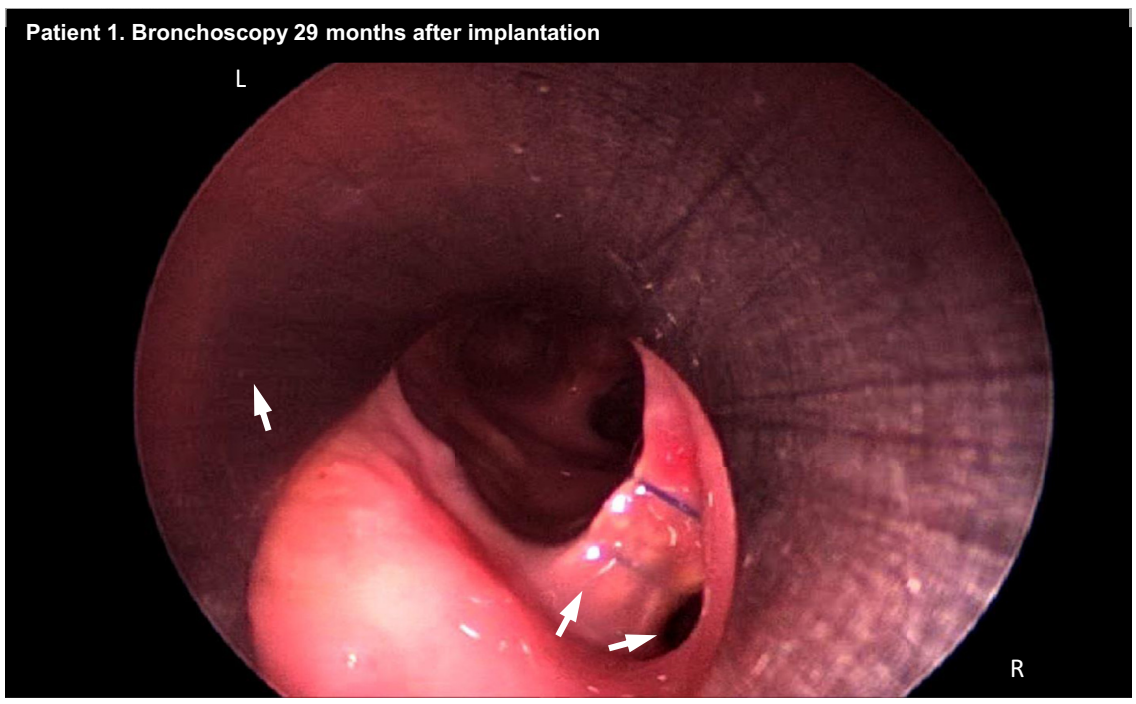

FIGURE E10. Patient 1. Bronchoscopy 29 months after implantation of the synthetic tracheal graft. Proximal graft anastomosis with large fistula. Arrows from left to right: inner wall of the bronchoscope, upper edge of the graft without mucosal lining, and the opening of a large proximal anastomotic tracheo-mediastinal-esophageal fistula (complete detachment of proximal graft anastomosis). 


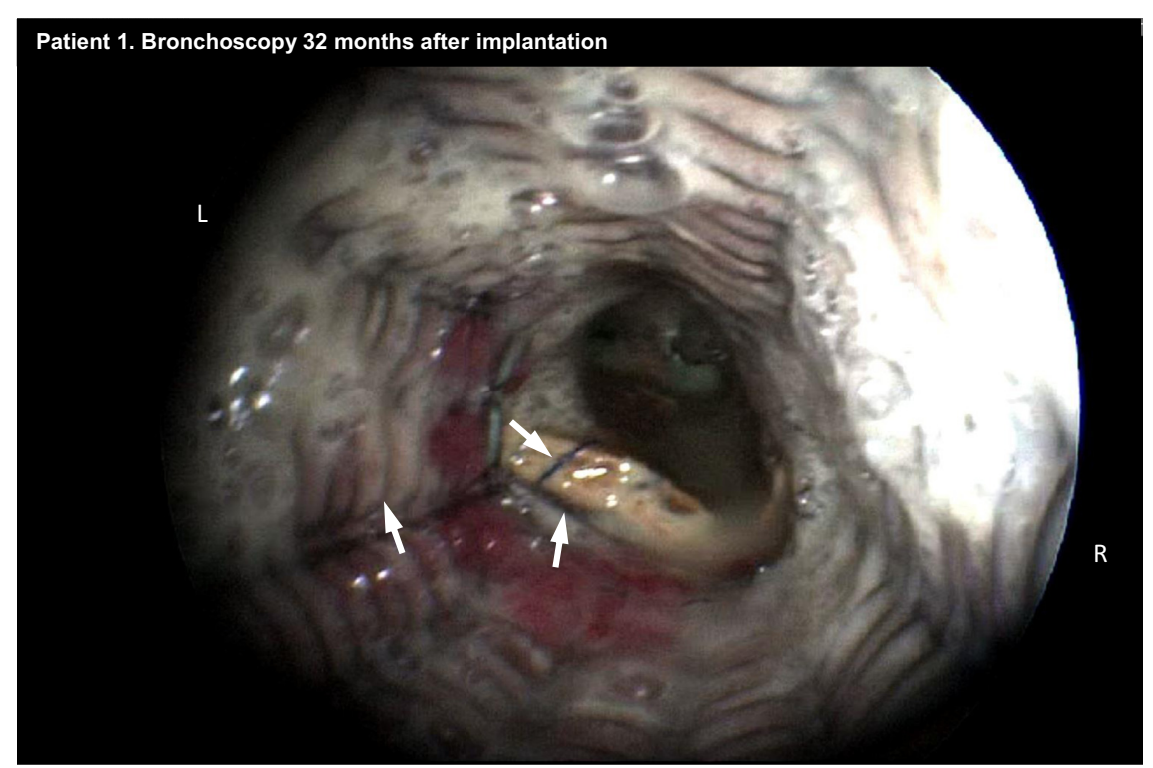

FIGURE E11. Patient 1. Bronchoscopy 32 months after implantation of the synthetic tracheal graft, 2 days before death. Proximal graft anastomosis with the detached graft. Massive leakage of fluid into the airway from the mediastinum and the esophagus by the completely detached proximal graft anastomosis. Arrows from left to right: airway stent dislocated from the proximal graft anastomosis up into the native trachea (inserted with the attempt to obliterate anastomosis leakage), proximal graft ending with exposed sutures and without mucosal lining, and complete circumferential detachment of the proximal graft ending from the native trachea (visible opening to the outer graft wall and mediastinum). 

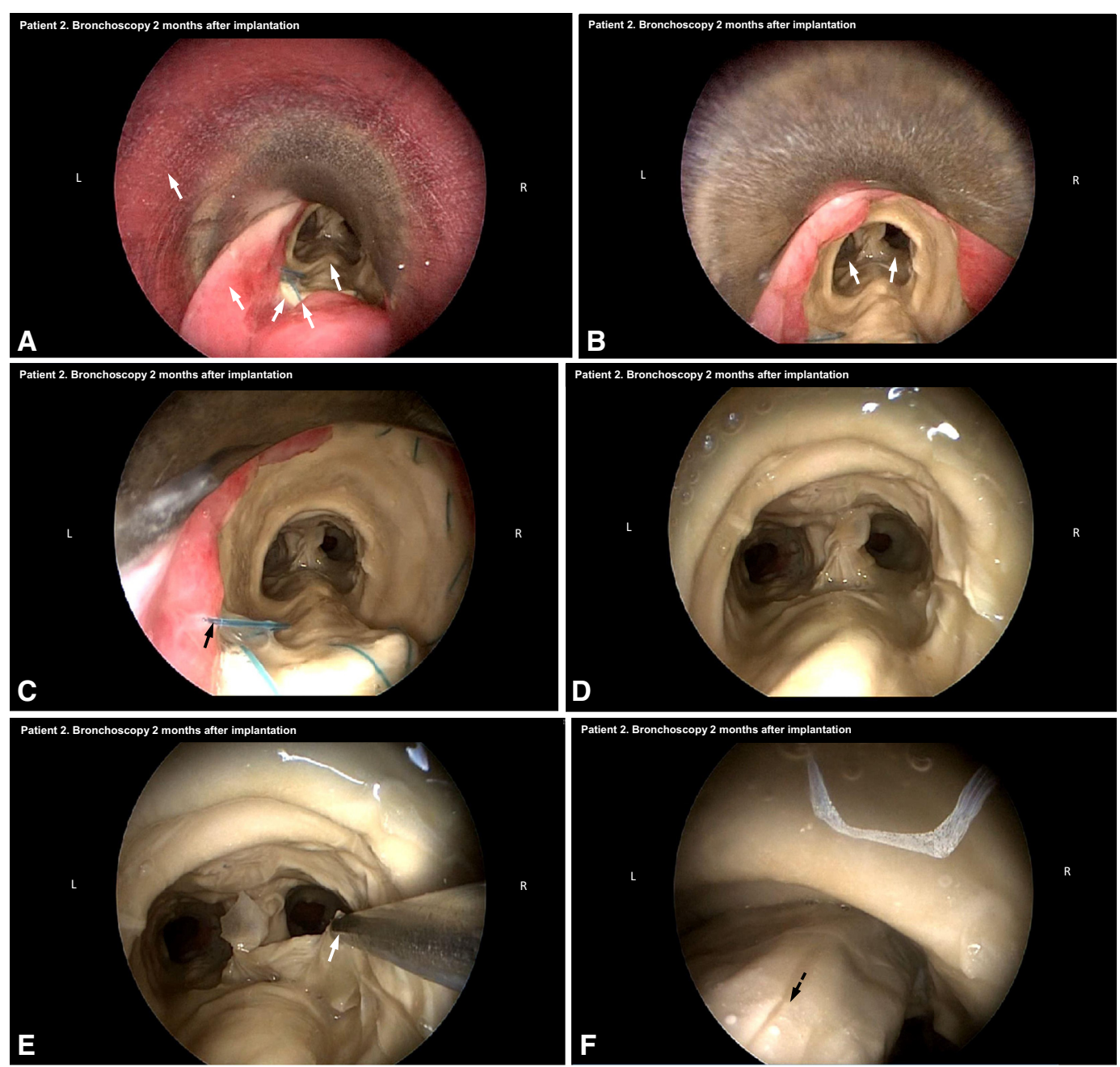

FIGURE E12. Patient 2. Bronchoscopy 2 months after implantation of the synthetic tracheal graft. A, Native trachea and the proximal part of the synthetic graft. Arrows from left to right: inner wall of the bronchoscope, native tracheal wall, proximal graft anastomosis, exposed sutures, and the graft without mucosal lining. B, Proximal synthetic graft. View advanced in distal direction into the graft. Left and right arrows: left and right distal graft departures. $\mathrm{C}$, Synthetic graft. Absence of mucosal lining on the graft 2 months after implantation. Arrow: exposed anastomotic suture. D, Inner wall of the synthetic graft with the left and right distal graft departures. View advanced in distal direction. The gray-yellow-colored layer covering the inner graft wall consists of a non-living layer of protein precipitate as a result from protein leakage into the graft. Protein precipitation does not imply establishment of mucosal lining. Under the layer of protein precipitate no mucosal lining was identified. E, Removal of protein precipitate from the inner wall of the synthetic graft. Arrow: bronchoscopy pincers gripping the layer of protein precipitate. F, Removal of protein precipitate from the inner wall of the synthetic graft. The whole frontal part of the graft's inner surface comes off when the layer of protein precipitate detaches as it is pulled further up to be removed. Dashed arrow: direction of the protein precipitate layer detaching (being pulled out) from the inner graft wall. G, Removal of protein precipitate from the inner wall of the synthetic graft. Dashed arrow: direction of the protein precipitate detaching from the inner graft wall as it is pulled further up to be removed. H, Removal of protein precipitate from the inner wall of the synthetic graft. Bronchoscopy pincers further removing the protein precipitate from the inner surface of the graft. Note the absence of vascularization. Under the layer of protein precipitate no mucosal lining was identified. I, Right distal graft anastomosis and the size mismatch between graft and native bronchus. Arrows from left to right: distal graft ending without mucosal lining 2 months after implantation, loose exposed anastomotic sutures, opening to the native right main bronchus, bleeding from manipulation of granulation tissue, and mucus. 

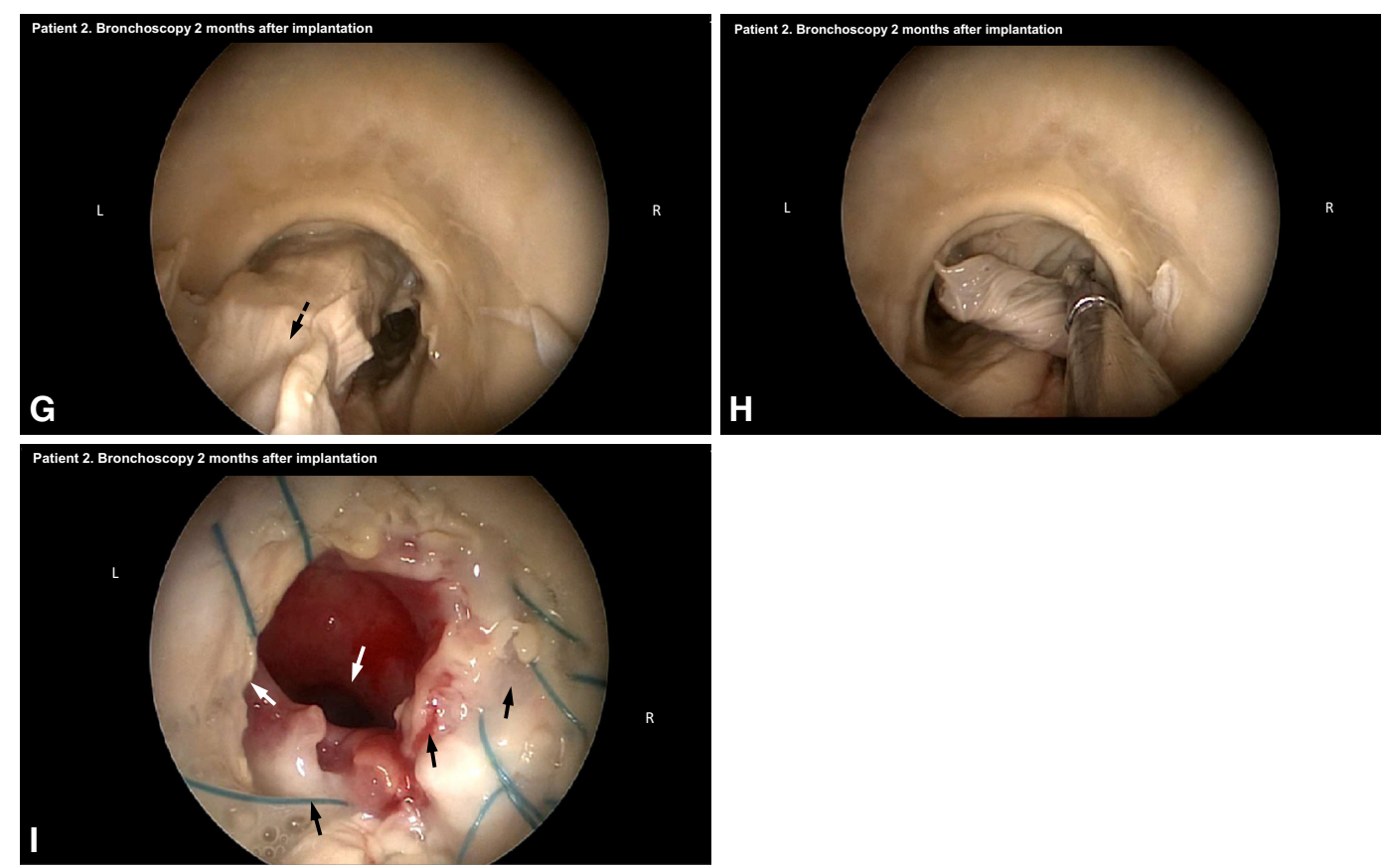

FIGURE E12. Continued 

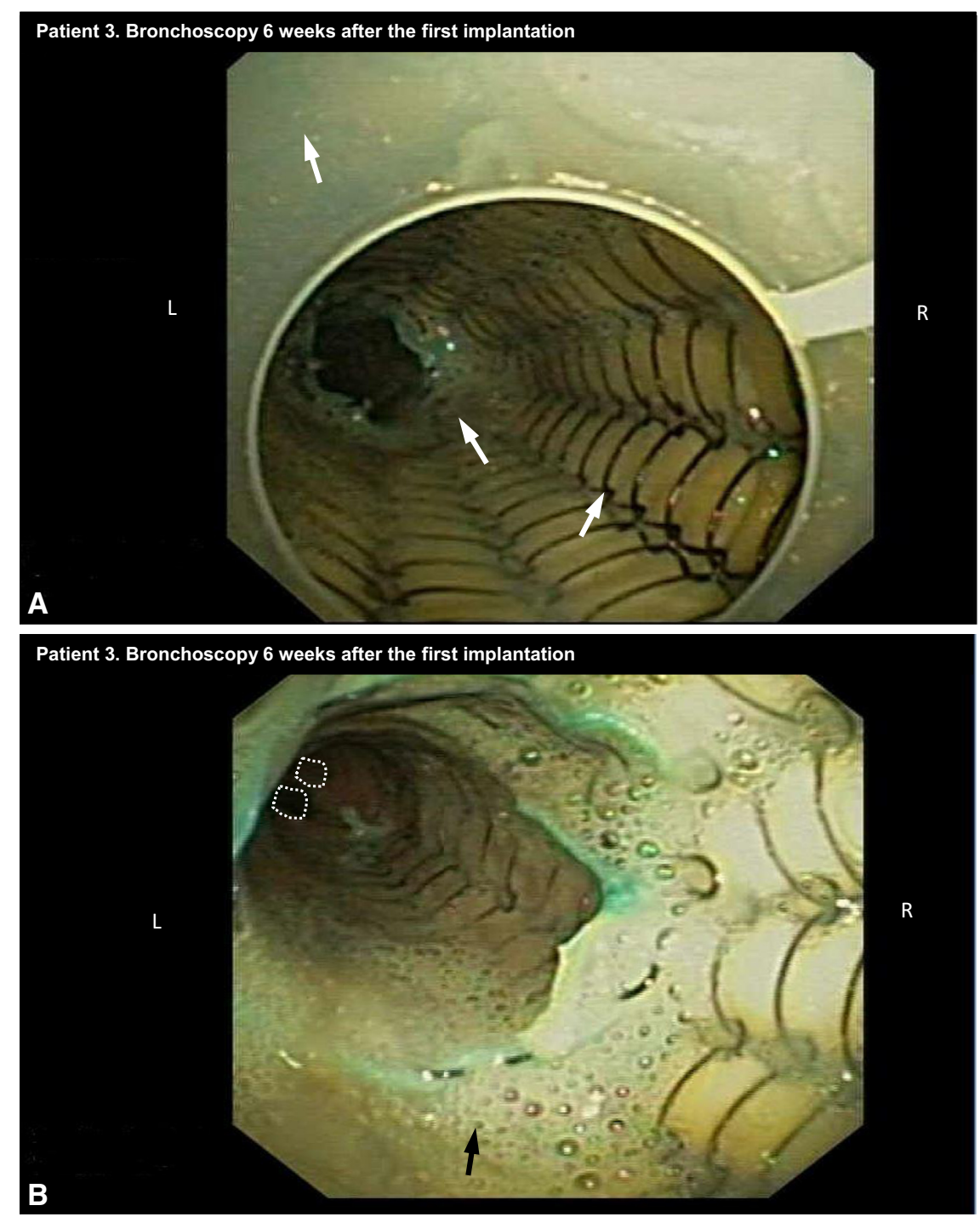

FIGURE E13. Patient 3. Bronchoscopy 6 weeks after implantation of the first synthetic tracheal graft. A, Inner wall of the synthetic graft with airway stents. Two airway stents cover the entire graft from the proximal graft anastomosis (outside of image) down to the native left upper and lower lobe departures (lobe carina). Inner graft wall (yellow colored) without mucosal lining seen between the stent wires. Arrows from left to right: inside of tracheal cannula, foamy mucus leaking into the airway through the fistula at the distal graft anastomosis, and airway stent. B, Distal airway stent in the native left main bronchus. Dotted circles: outlines the left upper (lower dotted circle) and lower (upper dotted circle) lobe departures. Arrow: significant leakage of foamy secretion passing into the airway through a large distal anastomotic fistula leading to massive aspiration and threating suffocation. 

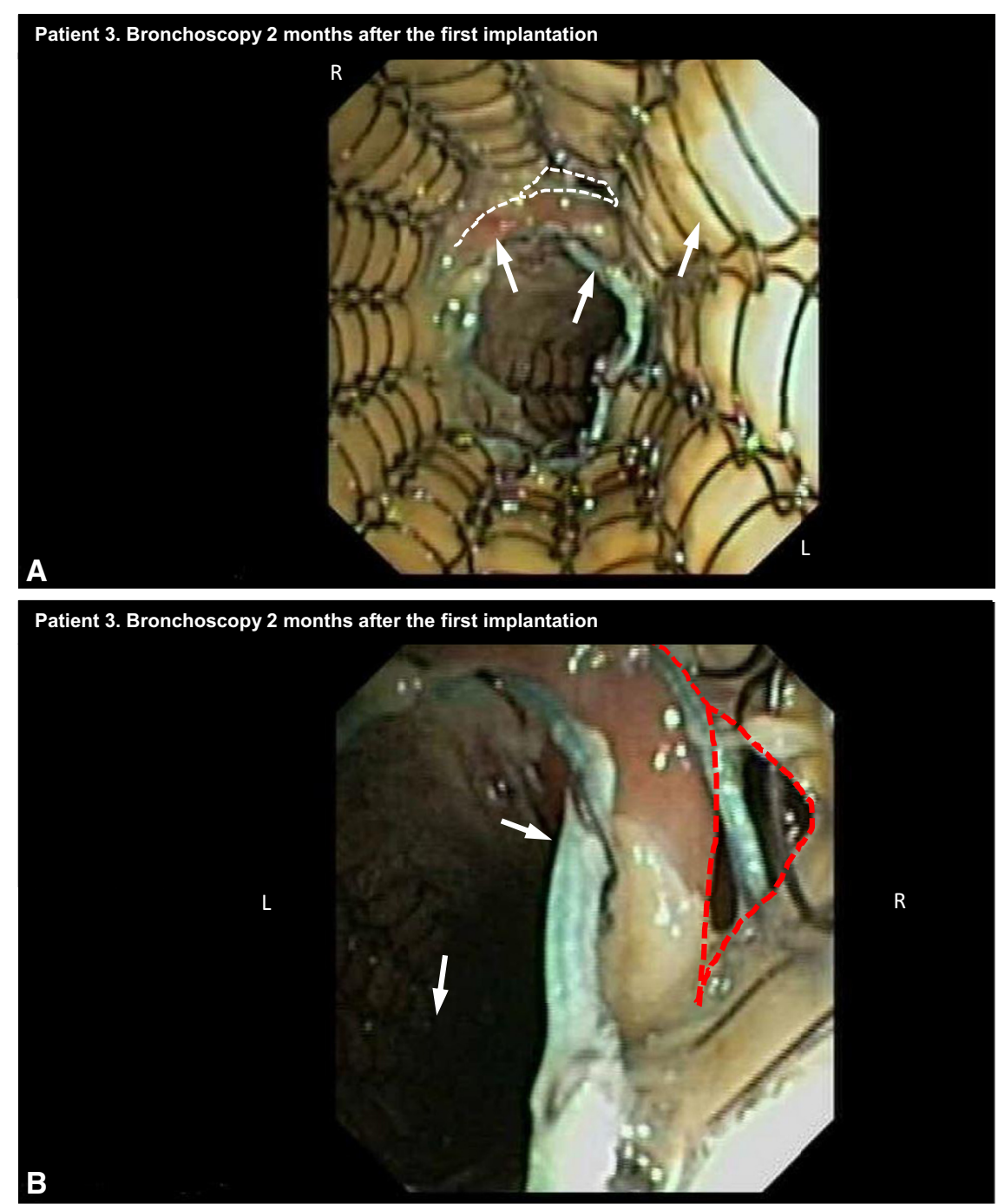

FIGURE E14. Patient 3. Bronchoscopy 2 months after implantation of the first synthetic tracheal graft. A, Synthetic graft with airway stents and the distal anastomotic fistula. Dashed line: outlines the distal anastomotic fistula (partly covered by mucus). Arrows from left to right: anastomotic granulation tissue, proximal ending of the distal airway stent (green-colored thread) inserted in the native left bronchus, and the yellow-white colored inner graft wall without mucosal lining. B, Distal graft anastomosis fistula. Left arrow: left main bronchus with airway stent to prevent it from collapsing and obstructing ventilation. Right arrow: proximal ending of distal airway stent (green-colored thread) in the native left bronchus. Dashed line: outlines the distal anastomotic fistula (partly covered by mucus). 


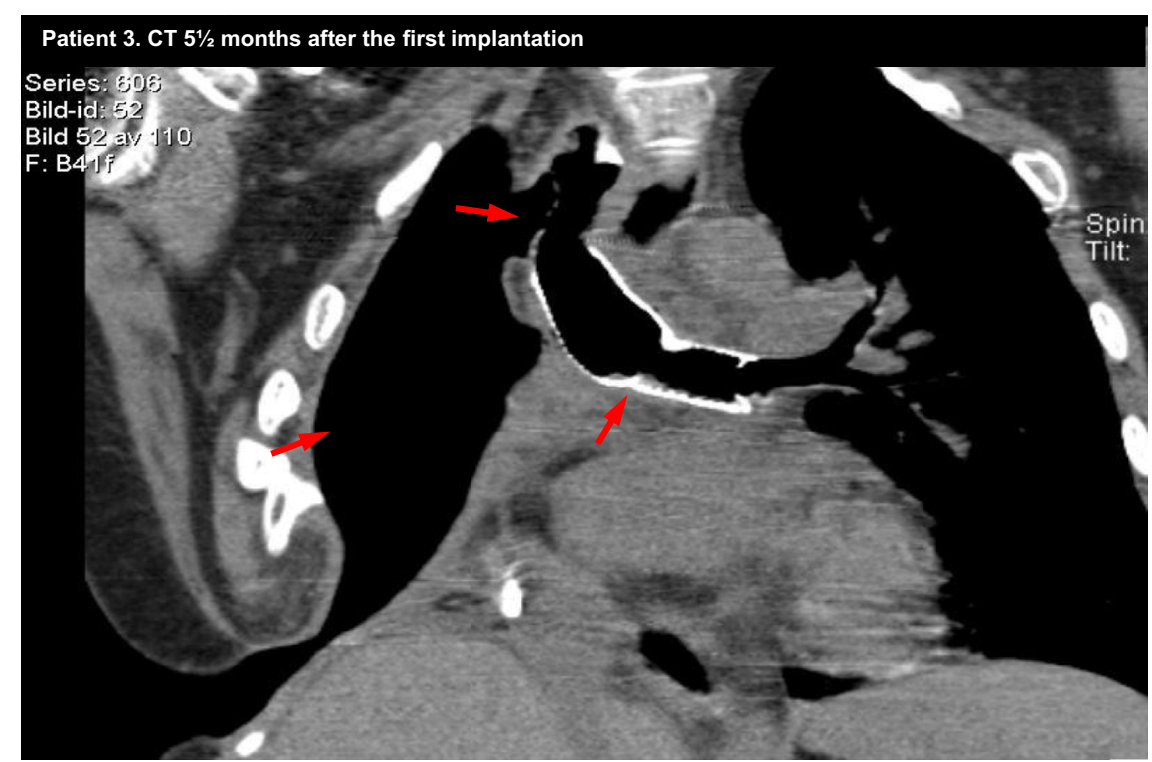

FIGURE E15. Patient 3. Computed tomography (CT) scan 5.5 months after implantation of the first synthetic tracheal graft. CT of the chest. Synthetic graft with airway stents and direct contact with ambient air. Arrows from left to right: open standing pleural cavity (colonized with bacteria), graft (with 2 inserted airway stents) directly exposed to bacteria and ambient air through the pleural cavity and the airway, and the distal anastomotic fistula. Note the deformed proximal part of the graft (material fatigue) with subsequent need for complementary stent insertion to prevent total graft collapse. 

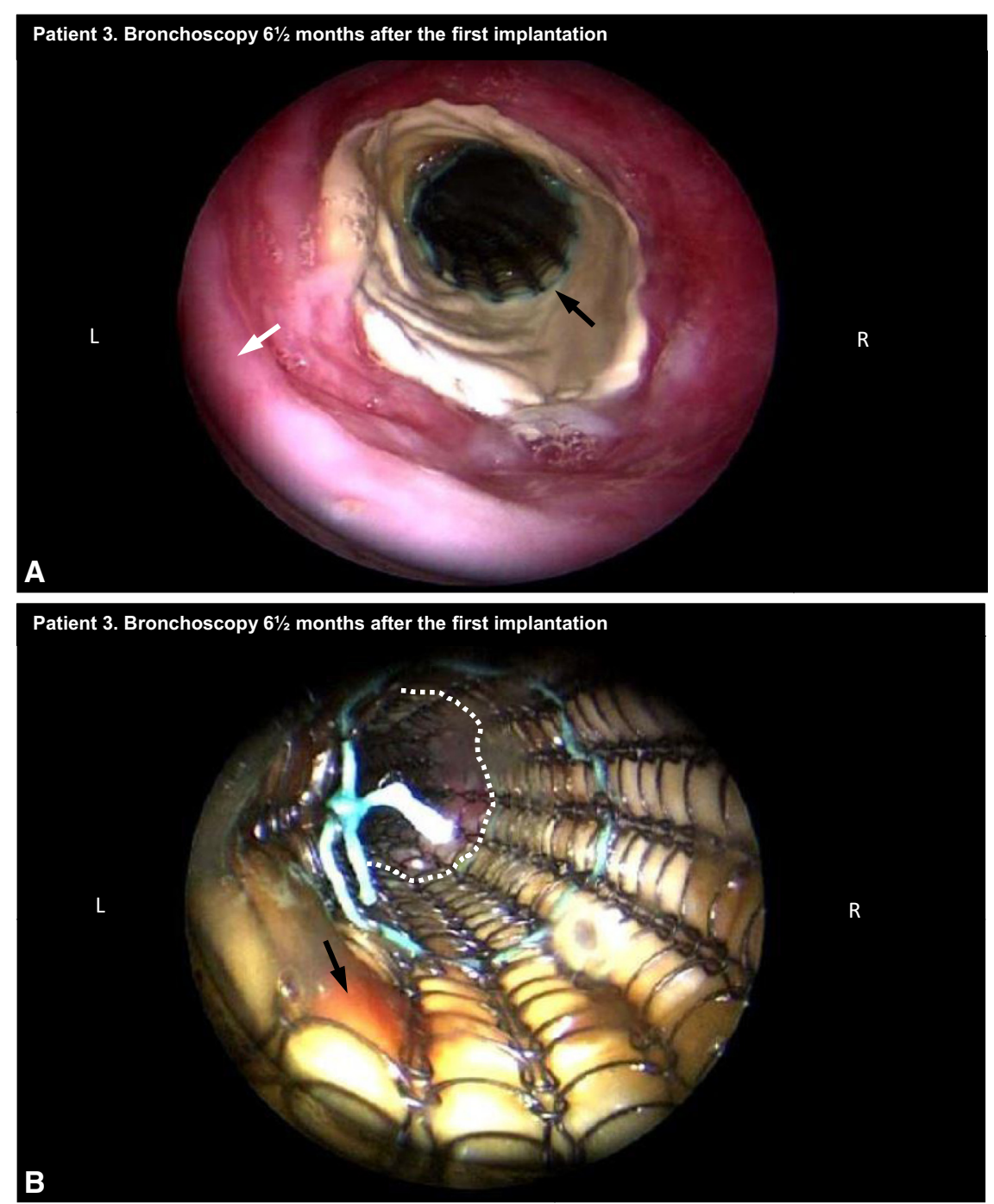

FIGURE E16. Patient 3. Bronchoscopy 6.5 months after implantation of the first synthetic tracheal graft. A, View from the native trachea towards the yellow-white colored graft without mucosal lining. Left arrow: native and normal vascularized tracheal tissue. Right arrow: airway stent inserted to prevent graft collapse due to material fatigue. Proximal part of the graft being oval shaped due to material fatigue. B, Inner wall of the synthetic graft and the proximal native left main bronchus. View advanced in distal direction. Arrow: blood tainted mucus (not vascularized mucosa) after resection of anastomotic granulation tissue. Dotted line: distal graft anastomosis. On the left (distal) side of the anastomosis the pink-colored mucosa of the native left bronchus. On the right (proximal) side the yellow-colored inner graft wall without mucosal lining. 


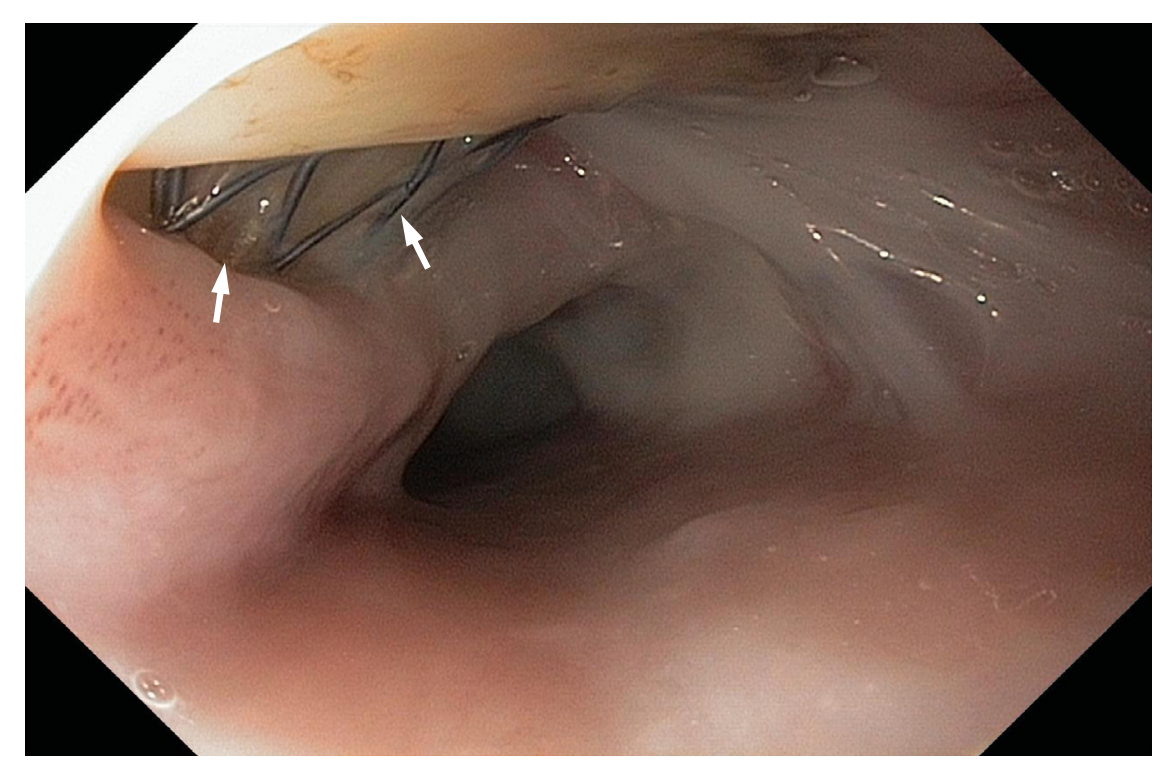

FIGURE E17. Patient 3. Esophagoscopy 8 months after implantation of the first synthetic tracheal graft. Tracheoesophageal fistulation. Left arrow: large tracheoesophageal fistula on the anterior esophageal wall. Right arrow: airway stent in the synthetic tracheal graft visible from the esophageal side. Airway stent covering the distal graft dehiscence between the graft and the native left main bronchus with an attempt (failed) to block leakage from the pleural cavity and the esophagus into the airway.

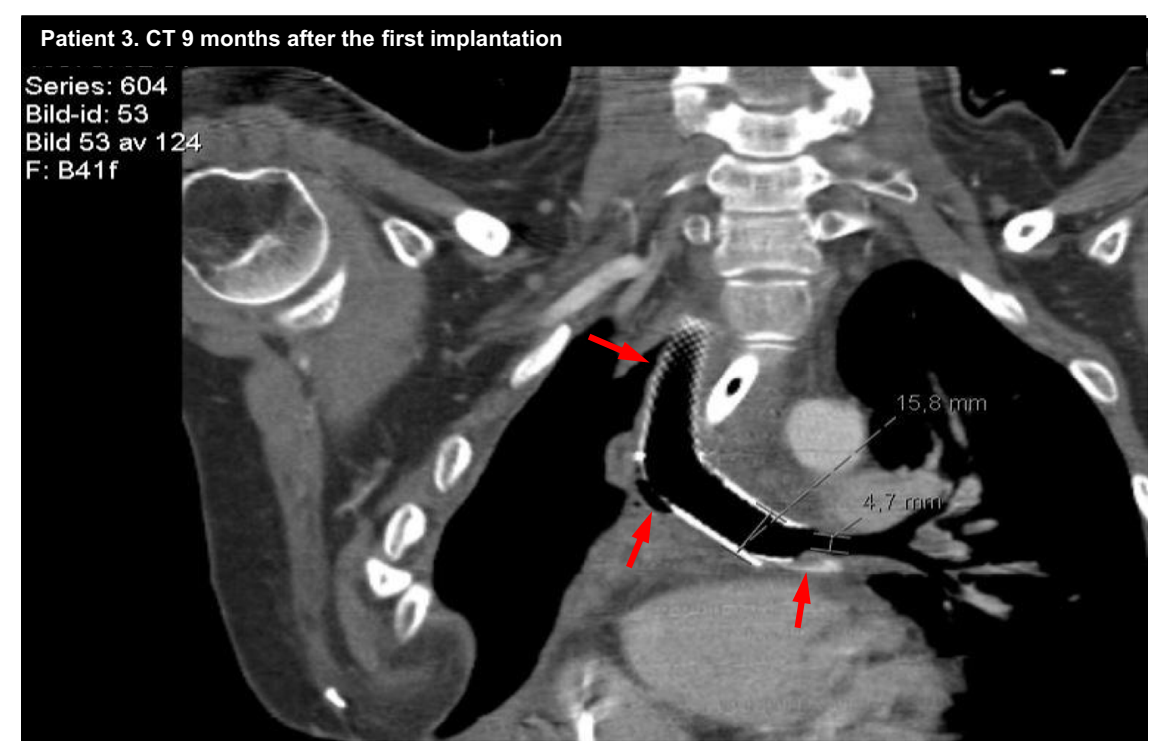

FIGURE E18. Patient 3. Computed tomography (CT) scan 9 months after implantation of the first synthetic tracheal graft. CT of the chest. Synthetic graft, anastomotic fistulae, and bronchus obstruction. Arrows from left to right: graft with air contact and 2 inserted airway stents, distal anastomotic fistula, and obstruction of the proximal part of the native bronchus by scaring and granulation tissue at the distal stent ending. 

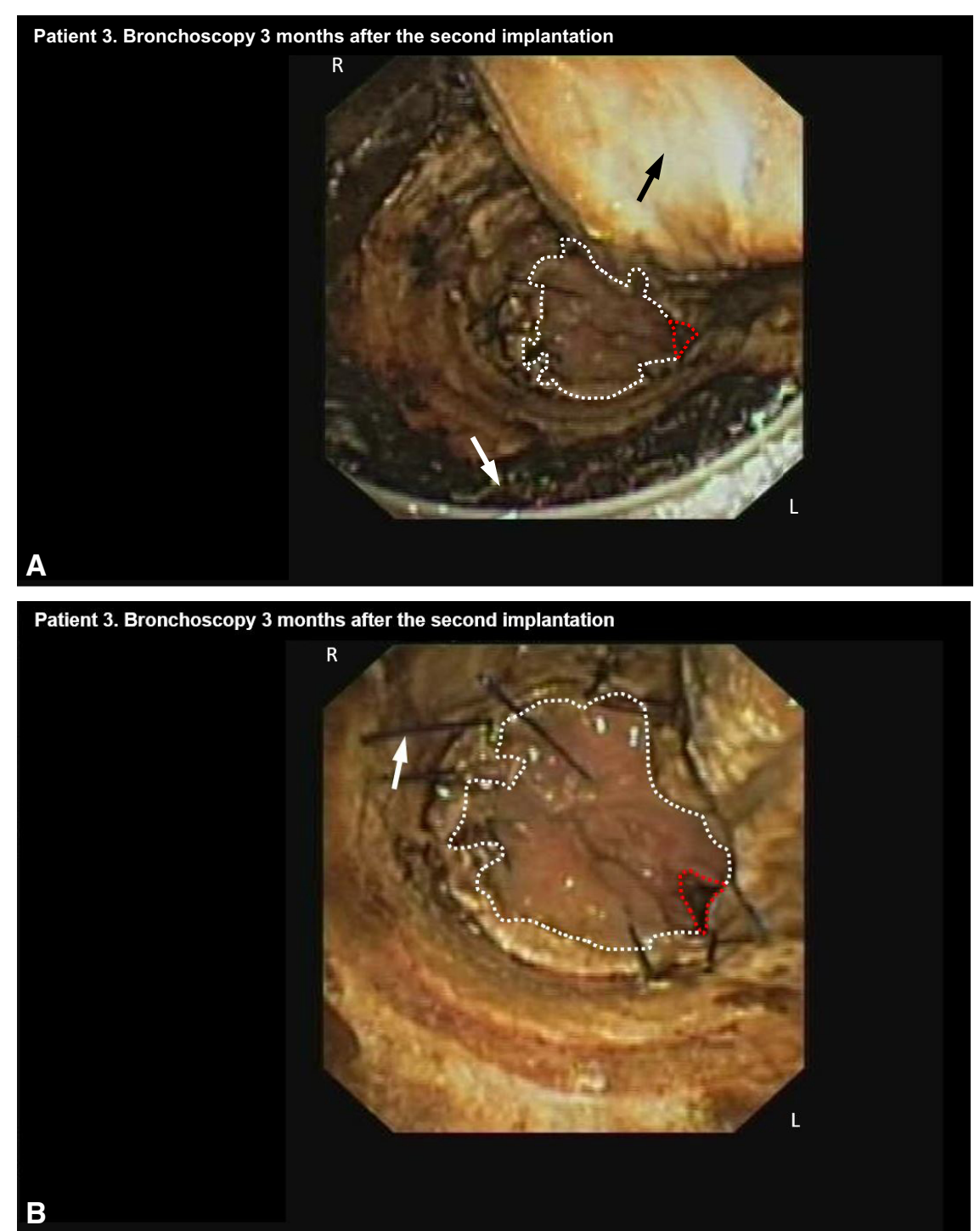

FIGURE E19. Patient 3. Bronchoscopy 3 months after implantation of the second synthetic tracheal graft. A, Inner wall of the synthetic graft with the native main bronchus opening. White dotted line: outlines the distal graft edge (distal anastomosis). Red dotted line: outlines the native left main bronchus opening. White arrow: inner graft wall with dried mucus and clots (bleeding secondary to resection of anastomotic granulation tissue) and no mucosal lining. Black arrow: posterior wall of the graft. Compare with Figure 1 presenting a normal and viable human tracheal inner wall. B, Synthetic graft with large size mismatch to the native left main bronchus opening. View advanced in distal direction into the graft without vascularization and mucosal lining. White dotted line: outlines the distal graft edge. Red dotted line: outlines the native left main bronchus opening. Arrow: exposed anastomotic sutures. 

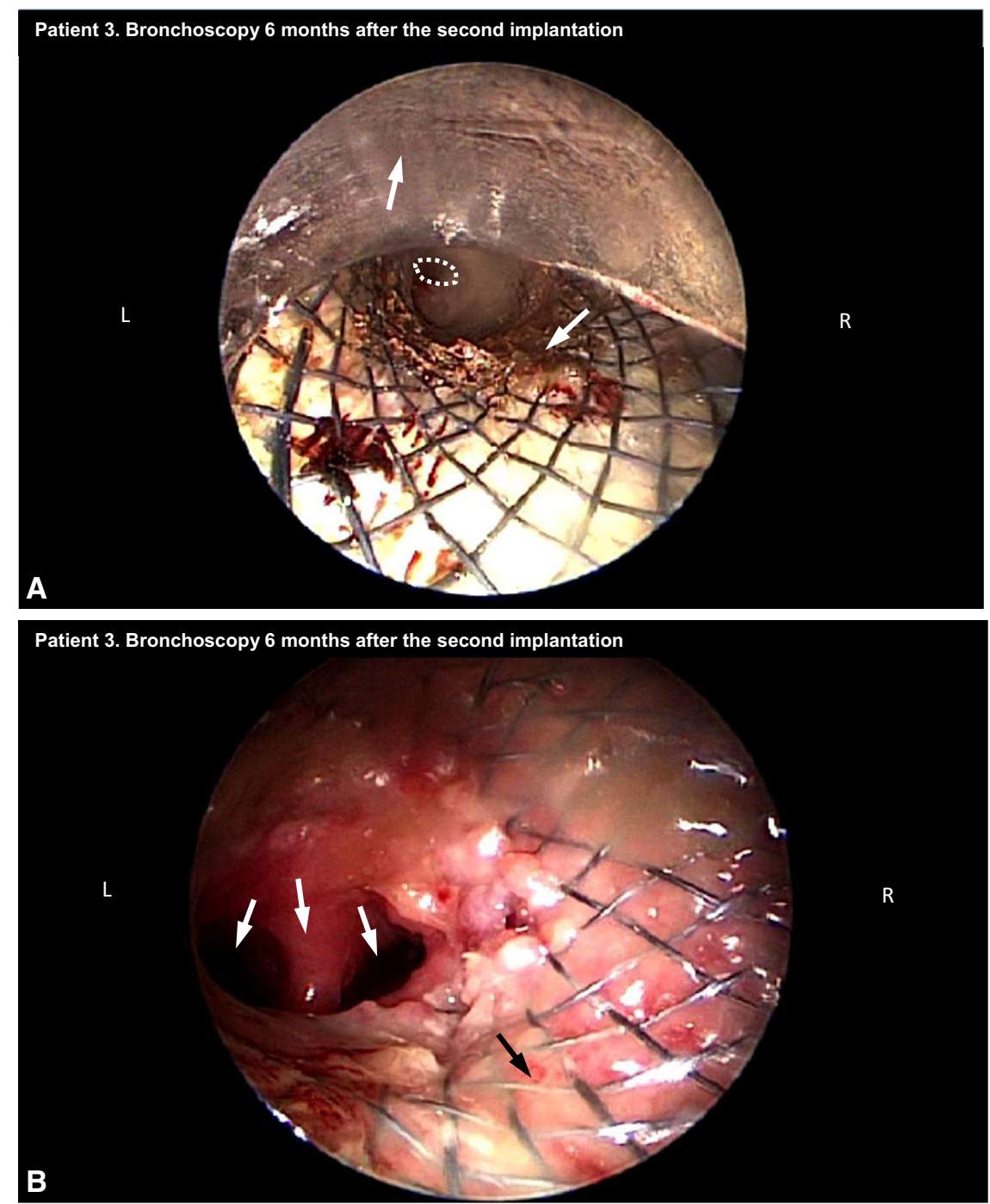

FIGURE E20. Patient 3. Bronchoscopy 6 months after implantation of the second synthetic tracheal graft. A, Distal part of the synthetic graft with airway stent. The yellow-white colored graft surface (seen through the stent wires) without mucosal lining. Left arrow: inner wall of the bronchoscope. Right arrow: dried blood clots from resection of anastomotic granulation tissue. White dotted line: outlines the left lung's lower lobe departure. B, Airway stent down to the upper and lower lobe departures of the left lung. View advanced in distal direction. The left main bronchus is stented down to the lobe carina (dividing the upper and lower lobe departures). Arrows from left to right: upper lobe departure, thickening of the lobe carina (scaring due to frequent stent interventions and removal of obstructive granulations), lower lobe departure, and airway stent covering the native and vascularized inner wall of the left distal main bronchus. 


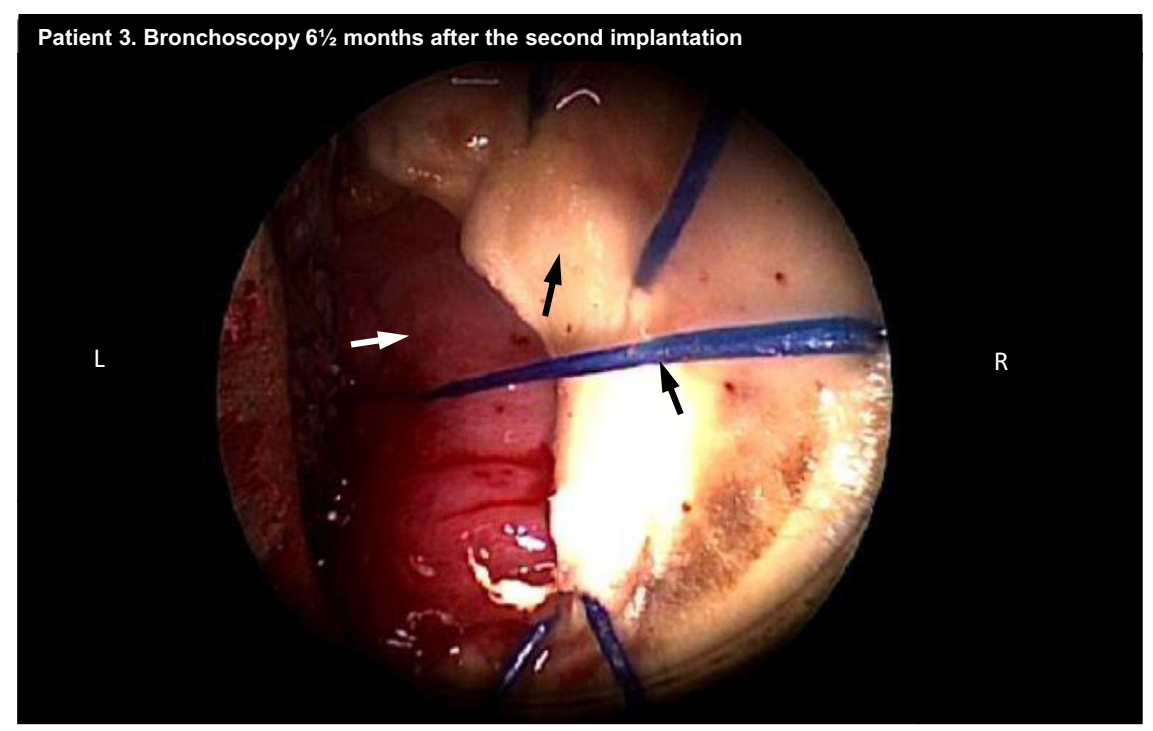

FIGURE E21. Patient 3. Bronchoscopy 6.5 months after implantation of the second synthetic tracheal graft. Distal graft anastomosis without integration of graft and native left main bronchus. Arrows from left to right: vascularized native left main bronchus, graft without mucosal lining 6.5 months after implantation, and exposed anastomotic sutures. 
TABLE E1. Adverse events and interventions of special interest*

\section{Adverse events and interventions}

Adverse events

Synthetic tracheal graft

Graft contamination by ambient air and oropharyngeal secretions

Bacterial and fungal colonization (chronic graft infection) $\ddagger$

Graft obstruction (stagnation of mucus, blood, pus)

Near-fatal suffocation event, $\mathrm{n}$

Graft collapse (synthetic material fatigue)

Graft anastomosis

Graft-mediastinal/-pleural fistula

Graft-esophageal fistula

Massive air leak§

Excessive formation of obstructive granulation tissue

Granulation bleeding (multiple life-threatening hemoptysis)

Anastomotic wound breakdown (graft dehiscence)

Dislocation of graft and graft-bronchial airway stent (numerous)

Infection, other than graft infection

Chronic infection in right-sided pneumectomy cavity $\dagger$

Pneumonia, numerous

Lung abscess

Mediastinitis

Sepsis

Chronic respiratory failure

Lung fibrosis secondary to chronic lung infection

Pulmonary bleeding

Thromboembolism (while on "regenerative boosting therapy")||

Pulmonary artery

Femoral artery

Jugular, subclavian and axillary veins

Extracorporeal membrane oxygenation pump thrombosis

Pericarditis

Hemolysis causing acute renal failure ||

Splenic infarction and bleeding

Laryngeal nerve paralysis

Multiple organ failure

Renal failure

Cachexia

Depression, hallucinations, despair

Hyperalgesia

Interventions secondary to graft dysfunction

Resection of obstructive anastomotic granulation tissue, numerous

Rigid bronchoscopy (airway stent evaluation and/or intervention), $\mathrm{n}$ ๆ

Chronic tracheostomy (for airway clearance)

Surgical tracheostomy revision

Daily scheduled and emergency flexible bronchoscopy, $\mathrm{n} \#$

Open standing thoracotomy, chronic chest drainage

Implantation of seconnd synthetic tracheal graft

Latissimus dorsi muscle flap at implantation of the second graft

Thoracic surgical reintervention (sternotomy)

Right-sided thoracoplasty (resection of ribs no. 2-8)

Surgical wound revision

Gastrosurgical intervention

Esophageal stenting, transection, esophagectomy, reconstruction**

Gastrectomy

Laparotomy

Percutaneous endoscopic gastrostomy $\dagger$

\begin{tabular}{|c|c|}
\hline+ & + \\
\hline+ & + \\
\hline . & + \\
\hline . & 40 \\
\hline & + \\
\hline & + \\
\hline . & + \\
\hline & + \\
\hline & + \\
\hline & + \\
\hline & + \\
\hline & + \\
\hline & + \\
\hline & + \\
\hline & + \\
\hline , & + \\
\hline & + \\
\hline 业 & .. \\
\hline & + \\
\hline & - \\
\hline & + \\
\hline & - \\
\hline & + \\
\hline & + \\
\hline & + \\
\hline & - \\
\hline & + \\
\hline & + \\
\hline & + \\
\hline & + \\
\hline & + \\
\hline & + \\
\hline & 56 \\
\hline & + \\
\hline & + \\
\hline & 7602 \\
\hline & + \\
\hline & 1 \\
\hline & 1 \\
\hline & 3 \\
\hline & 1 \\
\hline & 68 \\
\hline & 31 \\
\hline & + \\
\hline & + \\
\hline & + \\
\hline & + \\
\hline
\end{tabular}

(Continued) 
TABLE E1. Continued

\begin{tabular}{|c|c|c|c|}
\hline Adverse events and interventions & Patient 1 & Patient 2 & Patient $3 \dagger$ \\
\hline Ventilator support, $d$ & 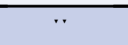 & $\cdot \cdot$ & 305 \\
\hline Sevoflurane sedation, wk & - & - & 5 \\
\hline Extracorporeal membrane oxygenation, $\mathrm{d}_{\uparrow \dagger} \dagger$ & - & - & 72 \\
\hline Splenectomy & - & - & + \\
\hline Hemodialysis, wk & .. & - & 7 \\
\hline
\end{tabular}

*Full listing of adverse events and interventions of special interest. Number of adverse events and intervenstions for all 3 patients are restricted to the time of hospitalization at Karolinska University Hospital and information provided in the medical records at Karolinska University Hospital, as complete data have not been accessible from the health care providers abroad. The plus sign $(+)$ denotes presence of the variable but not the number. The minus sign (-) denotes absence of the variable. Two mid-dots (") denote not available or not applicable data. $\dagger$ Data presented for patient 3 includes data after the first and second graft implantations. $\ddagger$ Multiresistant strepthomonas multi. (Stenothropomonas malthiphilia) in patient 1 and multiresistant Pseudomonas aeruginosa in patient 3. $§$ Massive air leak through the anastomotic fistula and out into room air by the open thoracotomy (open standing due to chronic thoracotomy cavity infection and pus accumulation). ||Thromboembolism while on the "regenerative boosting therapy." Patient 1 : pulmonary artery thrombus; patient 2: pulmonary artery, jugular, subclavian, and axillary vein thrombi; and patient 3 after the second graft implantation: femoral artery and extracorporeal membrane oxygenation pump thrombosis causing hemolysis and acute renal failure. $\uparrow$ Manufacturing of tailor-made airway stents (Leufen Medical, Berlin, Germany) for patient 3. \#Flexible bronchoscopies scheduled every fourth hour (6-15 per day) due to dysfunctional graft. From implantation of the first graft until the transfer to the thoracic transplant center after 3 years and 1 month. **Esophageal transection and subcutaneous colon interponate (patient 1). Esophagectomy and (failed) reconstruction by concomitant gastric pull up at the allogeneic trachea-left lung transplantation (patient 3). ††Patient 3 unable to orally intake food for nutrition for 4 years and 8 months due to tracheoesophageal fistulation, subsequent esophagectomy, and (failed) esophageal reconstruction. 拉Initiated due to insecure ventilation, massive anastomotic air leak, obstructing anastomotic granulation, and scar tissue. 